\title{
MIMO Zero-Forcing Performance Evaluation Using the Holonomic Gradient Method
}

\author{
Constantin Siriteanu, Akimichi Takemura, Satoshi Kuriki, Hyundong Shin, Christoph Koutschan
}

\begin{abstract}
For multiple-input multiple-output (MIMO) spatialmultiplexing transmission, zero-forcing detection (ZF) is appealing because of its low complexity. Our recent MIMO ZF performance analysis for Rician-Rayleigh fading, which is relevant in heterogeneous networks, has yielded for the $\mathrm{ZF}$ outage probability and ergodic capacity infinite-series expressions. Because they arose from expanding the confluent hypergeometric function ${ }_{1} F_{1}(\cdot, \cdot, \sigma)$ around $\mathbf{0}$, they do not converge numerically at realistically-high Rician $K$-factor values. Therefore, herein, we seek to take advantage of the fact that ${ }_{1} F_{1}(\cdot, \cdot, \sigma)$ satisfies a differential equation, i.e., it is a holonomic function. Holonomic functions can be computed by the holonomic gradient method (HGM), i.e., by numerically solving the satisfied differential equation. Thus, we first reveal that the moment generating function (m.g.f.) and probability density function (p.d.f.) of the ZF signal-to-noise ratio (SNR) are holonomic. Then, from the differential equation for ${ }_{1} F_{1}(\cdot, \cdot, \sigma)$, we deduce those satisfied by the SNR m.g.f. and p.d.f., and demonstrate that the HGM helps compute the p.d.f. accurately at practically-relevant values of $K$. Finally, numerical integration of the SNR p.d.f. produced by HGM yields accurate ZF outage probability and ergodic capacity results.
\end{abstract}

Index Terms-Holonomic gradient method, hypergeometric function, MIMO, Rayleigh-Rician fading, zero-forcing.

\section{INTRODUCTION}

\section{A. Background}

The performance of multiple-input multiple-output (MIMO) wireless communications systems has been attracting substantial interest [1] [2] [3] [4] [5]. Typically, its evaluation proceeds from expressions of performance measures - e.g., outage probability, ergodic capacity — derived based on statistical assumptions about the channel matrix [1] [2] [4] [6] [7] [8] [9] [10] [11] [12] [13].

For tractability, MIMO analyses have often assumed zeromean channel matrix, i.e., Rayleigh fading [2]. However, stateof-the-art channel measurements and models, e.g., WINNER II [14], have revealed nonzero-mean channel, i.e., Rician fading [2]. MIMO performance analysis for Rician fading is much more complicated than for Rayleigh fading. For linear interference-mitigation approaches such as zero-forcing

C. Siriteanu is with the Department of Information Systems Engineering, Graduate School of Information Science and Technology, Osaka University.

A. Takemura is with the Department of Mathematical Informatics, Graduate School of Information Science and Technology, University of Tokyo, Japan, and Japan Science and Technology Agency, CREST.

S. Kuriki is with the Institute of Statistical Mathematics, Tachikawa, Tokyo, Japan.

H. Shin is with the Department of Electronics and Radio Engineering, Kyung Hee University, South Korea.

C. Koutschan is with the Johann Radon Institute for Computational and Applied Mathematics, Austrian Academy of Sciences, Linz, Austria. detection $(\mathrm{ZF})$ and minimum mean-square error detection (MMSE) [15], the performance analysis of MIMO spatialmultiplexing transmission has been found tractable for RicianRayleigh mixtures [4] [12] (but not for general Rician fading).

ZF has been considered for WiMAX and LTE [16] and has recently been studied as relevant for distributed and large MIMO systems [5] [17] [9] [18] [10], mainly due to its low complexity. Nevertheless, although simple, for wellconditioned MIMO channel matrix, ZF approaches the performance of maximum-likelihood and minimum-error-rate (i.e., optimal) detection [19] [20] [21] [22]. Thus, the analysis of $\mathrm{ZF}$ performance for Rician fading has remained of interest see [11] [12] and references therein.

\section{B. Previous MIMO ZF Performance Analyses}

For perfectly-known uncorrelated Rayleigh fading channel, MIMO ZF performance was first characterized exactly in [23], by viewing $\mathrm{ZF}$ as the no-noise limit of optimum combining, i.e., MMSE [6, Remark 1]. Recently, though, it has been shown that performance measures for MMSE do not necessarily converge to those of ZF [24] [25] [26].

The results of [23] were extended to transmit-correlated fading in [7] [8] based on the fact that, given the channel matrix $\mathbf{H}$, the signal-to-noise ratio (SNR) for $\mathrm{ZF}$ is determined by matrix $\mathbf{H}^{\mathcal{H}} \mathbf{H}$, which is central-Wishart-distributed when $\mathbf{H}$ has zero mean and receive-correlation.

$\mathrm{ZF}$ has been studied for Rician fading, i.e., nonzero-mean $\mathbf{H}$, much less than for Rayleigh fading. This is because the analysis is complicated by the noncentral-Wishart distribution of $\mathbf{H}^{\mathcal{H}} \mathbf{H}$. Some approximation-based results for full-Rician fading have appeared for $\mathrm{ZF}$ in [11] and relevant references therein. For MMSE, approximate and exact analyses under Rician-Rayleigh fading mixtures appeared in [6] and [4], respectively.

Note that, although such fading mixtures have mainly been considered because they promote analysis tractability, they are nevertheless relevant in macrocells, microcells, and heterogeneous networks — see [4] [12] and references therein.

Thus, we have also analyzed $\mathrm{ZF}$ for such fading mixtures in [12] [13]. For the case when the intended stream undergoes Rician fading whereas the interfering streams undergo Rayleigh fading, [12] derived exact infinite-series expressions for $\mathrm{ZF}$ performance measures, and [13] proved their theoretical convergence.

The exact $\mathrm{ZF}$ analysis procedure we employed in [12] is as follows. First, we expressed, in [12, Eq. (31)], the moment generating function (m.g.f.) of the SNR for Rician-fading 
Stream 1 in terms of the confluent hypergeometric function ${ }_{1} F_{1}(\cdot, \cdot, \sigma)$ [27, Ch. 13]. Then, the well-known expansion of ${ }_{1} F_{1}(\cdot, \cdot, \sigma)$ around 0 from [27, Eq. (13.2.2), p. 322] yielded the infinite series for the SNR m.g.f. from [12, Eq. (37)]. Upon inverse-Laplace transformation, the latter yielded the infinite-series expression for the SNR probability density function (p.d.f.) from [12, Eq. (39)]. Finally, integration of this p.d.f. expression yielded the infinite-series expressions for ZF outage probability and ergodic capacity from [12, Eqs. (69), (71)].

\section{Limitation of Previous Performance Evaluation Approach}

As revealed in [12] [13], the computation of infinite series [12, Eqs. (39), (69), (71)] breaks down at much smaller Rician $K$-factor values than those considered realistic (e.g., averages of lognormal distributions proposed by WINNER II for $K$ in [14]). This is the consequence of the employed expansion of ${ }_{1} F_{1}(\cdot, \cdot, \sigma)$, whose own computation away from $\sigma=0$ is nontrivial: with increasing $\sigma$, numerical convergence is increasingly difficult (i.e., slow, resource-intensive) and eventually fails [28] [29] [30] [31].

Note that hypergeometric functions 11 and infinite-series expressions have been found to characterize the performance for many other MIMO techniques under many fading types [2] [4] [30] [31] [32]. Limitations in computing these infiniteseries for MIMO evaluation motivates our search herein for an alternative approach.

\section{New Approach and Contribution}

A seldom-considered approach for computing ${ }_{1} F_{1}(\cdot, \cdot, \sigma)$ follows from the fact that it satisfies, with respect to (w.r.t.) $\sigma$, a linear differential equation with polynomial coefficients [27, Eq. (13.2.1), p. 322], i.e., this function is holonomic [33, p. 334] [34, p. 7] [35, p. 140] [36. Section 6.4]. Any holonomic function can be computed at some $\sigma$ by numerically solving its differential equation starting from some $\sigma_{0}$ where the function is either known analytically or can be approximated accurately.

The computation of a holonomic function by numerically solving satisfied differential equations is known as the holonomic gradient method (HGM) [37] [38]. It has recently been applied in statistics to evaluating the normalizing constant of the Bingham distribution [37] and the cumulative distribution function (c.d.f.) of the dominant eigenvalue of a real-valued Wishart-distributed matrix [38], upon deriving relevant differential equations. To the best of our knowledge, the HGM has not yet been applied for MIMO performance evaluation, although MIMO performance measures have often been expressed in terms of holonomic special functions and ensuing infinite series [2] [31] [32] [28] [12] — see also [30] and references therein.

The current paper proposes the HGM-based evaluation of the exact MIMO ZF performance under Rician-Rayleigh fading. Starting from the ZF SNR m.g.f. expression derived in terms of ${ }_{1} F_{1}(\cdot, \cdot, \sigma)$ in [12. Eq. (31)] and the differential equation satisfied by ${ }_{1} F_{1}(\cdot, \cdot, \sigma)$ [27, Eq. (13.2.1), p. 322],

${ }^{1}$ Of both scalar and matrix arguments. we first deduce differential equations satisfied by the SNR m.g.f.. Their inverse-Laplace transformation yields differential equations satisfied by the SNR p.d.f..

These are shown to enable the accurate HGM-based computation of the SNR p.d.f. at practical $K$ values, by starting HGM from an initial p.d.f. value for $K \approx 0$, which can be computed accurately by truncating the available infiniteseries p.d.f. expression from [12, Eq. (39)]. Finally, numerical integration of the HGM output (i.e., the SNR p.d.f.) yields accurately, for the first time, the outage probability and ergodic capacity for MIMO ZF at $K$ values relevant to WINNER II.

The deduction of differential equations for MIMO performance measures and their HGM-based evaluation may lead to a new framework for MIMO analysis and evaluation under general fading. As the complexity of MIMO analyses seeking expressions (i.e., explicit representations) of performance measures has been increasing, an ability to derive - not only manually, as shown herein, but also by computer algebra, as shown in [39] - differential equations (i.e., implicit representations) for MIMO performance measures and to evaluate them by HGM may be a more general, more straightforward, and more effective alternative.

\section{E. Notation}

The notation defined below follows closely that from [12]. Thus, scalars, vectors, and matrices are represented with lowercase italics, lowercase boldface, and uppercase boldface, respectively, e.g., $a, \mathbf{h}$, and $\mathbf{H}$; superscripts ${ }^{\mathcal{T}}$ and $\cdot \mathcal{H}$ stand for transpose and Hermitian (i.e., complex-conjugate) transpose; $[\cdot]_{i, j}$ indicates the $i, j$ th element of a matrix; $\|\mathbf{H}\|^{2}=\sum_{i}^{N_{\mathrm{R}}} \sum_{j}^{N_{\mathrm{T}}}\left|[\mathbf{H}]_{i, j}\right|^{2}$ is the squared Frobenius norm of $N_{\mathrm{R}} \times N_{\mathrm{T}}$ matrix $\mathbf{H} ; \propto$ stands for 'proportional to'; subscripts $\cdot d$ and $\cdot r$ identify, respectively, the deterministic and random components; subscript $\cdot_{n}$ indicates a normalized variable; $\mathbb{E}\{\cdot\}$ denotes statistical average; $(N)_{n}$ is the Pochhammer symbol, i.e., $(N)_{0}=1$ and $(N)_{n}=N(N+1) \ldots(N+n-1), \forall n>1$ [27, p. xiv], and ${ }_{1} F_{1}(\cdot ; \cdot ; \cdot)$ is the confluent hypergeometric function [27, Eq. (13.2.2), p. 322].

\section{F. Paper Organization}

Section II describes the MIMO signal, noise, and channel models. Section III introduces the ZF SNR m.g.f. and p.d.f. infinite-series expressions derived in [12], and discusses difficulties encountered in the computation of the infinite series for the p.d.f. and ensuing performance measures. Section IV defines holonomic functions and deduces from their properties that the SNR m.g.f. and p.d.f. are holonomic. This justifies our search in Section $\mathrm{V}$ for the differential equations they satisfy. These differential equations are then exploited using the HGM to produce the numerical results shown and discussed in Section VI Finally, Section VII discusses other possible HGM applications in MIMO evaluation.

\section{Signal, Noise, And FAding Models}

Herein, the signal, noise, and channel models and assumptions follow closely the ones from [12]. Thus, we consider 
uncoded MIMO spatial-multiplexing over a frequency-flat fading channel and assume that there are $N_{\mathrm{T}}$ and $N_{\mathrm{R}}$ antenna elements at the transmitter(s) and receiver, respectively, with $N_{\mathrm{T}} \leq N_{\mathrm{R}}$. Let us denote the number of degrees of freedom as

$$
N=N_{\mathrm{R}}-N_{\mathrm{T}}+1 .
$$

Letting $\mathbf{x}=\left[x_{1} x_{2} \cdots x_{N_{\mathrm{T}}}\right]^{\mathcal{T}}$ denote the $N_{\mathrm{T}} \times 1$ zero-mean transmit-symbol vector with $\mathbb{E}\left\{\mathbf{x x}^{\mathcal{H}}\right\}=\mathbf{I}_{N_{\mathrm{T}}}$, the $N_{\mathrm{R}} \times 1$ vector with the received signals can be represented as [1, Eq. (8)] [12. Eq. (1)]:

$\mathbf{r}=\sqrt{\frac{E_{\mathrm{s}}}{N_{\mathrm{T}}}} \mathbf{H} \mathbf{x}+\mathbf{v}=\sqrt{\frac{E_{\mathrm{s}}}{N_{\mathrm{T}}}} \mathbf{h}_{1} x_{1}+\sqrt{\frac{E_{\mathrm{s}}}{N_{\mathrm{T}}}} \sum_{k=2}^{N_{\mathrm{T}}} \mathbf{h}_{k} x_{k}+\mathbf{v}$.

Above, $E_{\mathrm{s}} / N_{\mathrm{T}}$ represents the energy transmitted per symbol (i.e., per antenna), so that $E_{\mathrm{s}}$ is the energy transmitted per channel use. The additive noise vector $\mathbf{v}$ is zero-mean, uncorrelated, circularly-symmetric complex-valued Gaussian [1] with variance $N_{0}$ per dimension. We will also employ its normalized version $\mathbf{v}_{\mathrm{n}}=\mathbf{v} / \sqrt{N_{0}}$. We shall employ the persymbol input SNR, defined as

$$
\Gamma_{\mathrm{s}}=\frac{E_{\mathrm{s}}}{N_{0}} \frac{1}{N_{\mathrm{T}}},
$$

as well as the per-bit input SNR, which, for a modulation constellation with $M$ symbols (e.g., MPSK), is defined as

$$
\Gamma_{\mathrm{b}}=\frac{\Gamma_{\mathrm{s}}}{\log _{2} M} .
$$

Then, $\mathbf{H}=\left(\begin{array}{llll}\mathbf{h}_{1} & \mathbf{h}_{2} & \ldots & \mathbf{h}_{N_{\mathrm{T}}}\end{array}\right)$ is the $N_{\mathrm{R}} \times N_{\mathrm{T}}$ complexGaussian channel matrix. Vector $\mathbf{h}_{k}$ comprises the channel factors between transmit-antenna $k$ and all receive-antennas. The deterministic (i.e., mean) and random components of $\mathbf{H}$ are denoted as $\mathbf{H}_{\mathrm{d}}=\left(\begin{array}{lllll}\mathbf{h}_{\mathrm{d}, 1} & \mathbf{h}_{\mathrm{d}, 2} & \ldots & \mathbf{h}_{\mathrm{d}, N_{\mathrm{T}}}\end{array}\right)$ and $\mathbf{H}_{\mathrm{r}}=$ $\left(\begin{array}{llll}\mathbf{h}_{\mathrm{r}, 1} & \mathbf{h}_{\mathrm{r}, 2} & \ldots & \mathbf{h}_{\mathrm{r}, N_{\mathrm{T}}}\end{array}\right)$, respectively, so that $\mathbf{H}=\mathbf{H}_{\mathrm{d}}+\mathbf{H}_{\mathrm{r}}$. If $\left[\mathbf{H}_{\mathrm{d}}\right]_{i, j}=0$ then $\left|[\mathbf{H}]_{i, j}\right|$ has a Rayleigh distribution; otherwise, $\left|[\mathbf{H}]_{i, j}\right|$ has a Rician distribution [2]. Typically, the channel matrix for Rician fading is written as [1]

$$
\mathbf{H}=\mathbf{H}_{\mathrm{d}}+\mathbf{H}_{\mathrm{r}}=\sqrt{\frac{K}{K+1}} \mathbf{H}_{\mathrm{d}, \mathrm{n}}+\sqrt{\frac{1}{K+1}} \mathbf{H}_{\mathrm{r}, \mathrm{n}},
$$

where, for normalization purposes [40], it is assumed that

$$
\left\|\mathbf{H}_{\mathrm{d}, \mathrm{n}}\right\|^{2}=\mathbb{E}\left\{\left\|\left[\mathbf{H}_{\mathrm{r}, \mathrm{n}}\right]\right\|^{2}\right\}=N_{\mathrm{T}} N_{\mathrm{R}},
$$

so that $\mathbb{E}\left\{\|\mathbf{H}\|^{2}\right\}=N_{\mathrm{T}} N_{\mathrm{R}}$. Power ratio

$$
K=\frac{\left\|\mathbf{H}_{\mathrm{d}}\right\|^{2}}{\mathbb{E}\left\{\left\|\mathbf{H}_{\mathrm{r}}\right\|^{2}\right\}}=\frac{\frac{K}{K+1}\left\|\mathbf{H}_{\mathrm{d}, \mathrm{n}}\right\|^{2}}{\frac{1}{K+1} \mathbb{E}\left\{\left\|\mathbf{H}_{\mathrm{r}, \mathrm{n}}\right\|^{2}\right\}}
$$

is the Rician $K$-factor: $K=0$ yields Rayleigh fading for all elements of $\mathbf{H} ; K \neq 0$ yields Rician fading if $\mathbf{H}_{\mathrm{d}, \mathrm{n}} \neq \mathbf{0}$.

In [12], we partitioned into the column with the fading gains that affect the intended stream, e.g., Stream 1, and the matrix columns with the fading gains that affect the interfering streams, i.e.,

$$
\mathbf{H}=\left(\begin{array}{ll}
\mathbf{h}_{1} & \mathbf{H}_{2}
\end{array}\right)=\left(\begin{array}{ll}
\mathbf{h}_{\mathrm{d}, 1} & \mathbf{H}_{\mathrm{d}, 2}
\end{array}\right)+\left(\begin{array}{ll}
\mathbf{h}_{\mathrm{r}, 1} & \mathbf{H}_{\mathrm{r}, 2}
\end{array}\right),
$$

and assumed, for analysis tractability, that $\mathbf{h}_{\mathrm{d}, 1} \neq \mathbf{0}$ and $\mathbf{H}_{\mathrm{d}, 2}=\mathbf{0}$, i.e., Rician-Rayleigh fading. Then, we can write

$$
\begin{aligned}
\left\|\mathbf{h}_{\mathrm{d}, 1}\right\|^{2} & =\left\|\left(\mathbf{h}_{\mathrm{d}, 1} \quad \mathbf{0}_{N_{\mathrm{R}} \times\left(N_{\mathrm{T}}-1\right)}\right)\right\|^{2}=\left\|\mathbf{H}_{\mathrm{d}}\right\|^{2} \\
& =\frac{K}{K+1} N_{\mathrm{R}} N_{\mathrm{T}} .
\end{aligned}
$$

As in [7] [8], for tractability, we assume zero receivecorrelation and we allow for nonzero transmit-correlation whereby all conjugate-transposed rows of $\mathbf{H}_{\mathrm{r}, \mathrm{n}}$ have distribution $\mathcal{C N}\left(\mathbf{0}, \mathbf{R}_{\mathrm{T}}\right)$. Consequently, all conjugate-transposed rows of $\mathbf{H}_{\mathrm{r}}$ have distribution $\mathcal{C N}\left(\mathbf{0}, \mathbf{R}_{\mathrm{T}, K}=\frac{1}{K+1} \mathbf{R}_{\mathrm{T}}\right)$.

It can be shown that normalization $\mathbb{E}\left\{\left\|\left[\mathbf{H}_{\mathrm{r}, \mathrm{n}}\right]\right\|^{2}\right\}=N_{\mathrm{T}} N_{\mathrm{R}}$ is equivalent with

$$
\sum_{i=1}^{N_{\mathrm{T}}}\left[\mathbf{R}_{\mathrm{T}}\right]_{i, i}=N_{\mathrm{T}}
$$

Because the diagonal elements of $\mathbf{R}_{\mathrm{T}}$ need not be equal, our analysis applies also for distributed transmitters. Nevertheless, for simplicity, numerical results are shown herein only for the case $\left[\mathbf{R}_{\mathrm{T}}\right]_{i, i}=1, \forall i$, i.e., for collocated transmitters.

The elements of $\mathbf{R}_{\mathrm{T}}$ can be computed from the azimuth spread (AS) as shown in [11, Section VI.A] for WINNER II, i.e., Laplacian, power azimuth spectrum. Note that WINNER II has modeled both $K$ (in $\mathrm{dB}$ ) and AS (in degrees) as random variables with scenario-dependent lognormal distributions [14]. Herein, we show results for $K$ and AS set to their averages for WINNER II indoor scenario A1.

\section{INFINITE-SERIES EXPRESSIONS FOR MIMO ZF SNR M.G.F. AND P.D.F.}

\section{A. MIMO ZF and Its SNR M.G.F. for Rician-Rayleigh Fading}

For the received-signal vector from (2), ZF means identifying the closest modulation constellation symbol for each element of the vector [1, Eq. (22)]

$$
\sqrt{\frac{N_{\mathrm{T}}}{E_{\mathrm{s}}}}\left[\mathbf{H}^{\mathcal{H}} \mathbf{H}\right]^{-1} \mathbf{H}^{\mathcal{H}} \mathbf{r}=\mathbf{x}+\frac{1}{\sqrt{\Gamma_{\mathrm{s}}}}\left[\mathbf{H}^{\mathcal{H}} \mathbf{H}\right]^{-1} \mathbf{H}^{\mathcal{H}} \mathbf{v}_{\mathbf{n}} .
$$

Then, the ZF SNR for Stream 1 is given by [23]

$$
\gamma_{1}=\frac{\Gamma_{\mathrm{s}}}{\left[\left(\mathbf{H}^{\mathcal{H}} \mathbf{H}\right)^{-1}\right]_{1,1}} .
$$

Its m.g.f. is defined as [2. Eq. (1.2)]

$$
M_{\gamma_{1}}(s)=\mathbb{E}\left\{e^{s \gamma_{1}}\right\}=\int_{0}^{\infty} e^{s t} p_{\gamma_{1}}(t) \mathrm{d} t,
$$

where $\mathbb{E}\{\cdot\}$ stands for mean, and $p_{\gamma_{1}}(t, a)$ is the p.d.f. of $\gamma_{1}$. Thus, the m.g.f. is related to the Laplace transform [27. Eq. (1.14.17)] $L_{\gamma_{1}}(s, a)$ of the p.d.f. by the sign change

$$
M_{\gamma_{1}}(-s)=L_{\gamma_{1}}(s)=\int_{0}^{\infty} e^{-s t} p_{\gamma_{1}}(t) \mathrm{d} t .
$$

If we define as in [12, Eqs. (18), (23)], respectively,

$$
\begin{aligned}
\Gamma_{1} & =\frac{\Gamma_{\mathrm{s}}}{\left[\mathbf{R}_{\mathrm{T}, K}^{-1}\right]_{1,1}} \propto \frac{\Gamma_{\mathrm{s}}}{K+1}, \\
a & =\left[\mathbf{R}_{\mathrm{T}, K}^{-1}\right]_{1,1}\left\|\mathbf{h}_{\mathrm{d}, 1}\right\|^{2} \propto K N_{\mathrm{R}} N_{\mathrm{T}},
\end{aligned}
$$


then, for MIMO ZF under Rician-Rayleigh fading, we can write the following exact expression for the m.g.f. of the SNR of the Rician-fading Stream 1, from [12, Eq. (31)]:

$$
M_{\gamma_{1}}(s, a)=\frac{1}{\left(1-\Gamma_{1} s\right)^{N}}{ }_{1} F_{1}\left(N ; N_{\mathrm{R}} ; a \frac{\Gamma_{1} s}{1-\Gamma_{1} s}\right),
$$

where ${ }_{1} F_{1}\left(N ; N_{\mathrm{R}} ; \sigma\right)$ is the confluent hypergeometric function of scalar argument $\sigma$ [27, Ch. 13].

For the SNR m.g.f. in (17) we have added $a \propto K$ as variable, and, hereafter, also for the SNR p.d.f., because for p.d.f. evaluation we shall apply HGM, which requires differential equations w.r.t. $a-$ they are deduced from (17) further below, in Section V-D Hereafter in this Section, we show the infinite series obtained for the SNR p.d.f. in [12], and explain its numerical convergence difficulties with increasing $a$ (i.e., $K$ ).

\section{B. Infinite Series for SNR M.G.F. and P.D.F. for Rician- Rayleigh Fading}

The confluent hypergeometric function is well-known to have the infinite-series expansion [27, Eq. (13.2.2), p. 322]

$$
{ }_{1} F_{1}\left(N ; N_{\mathrm{R}} ; \sigma\right)=\sum_{n=0}^{\infty} \frac{(N)_{n}}{\left(N_{\mathrm{R}}\right)_{n}} \frac{\sigma^{n}}{n !}=\sum_{n=0}^{\infty} A_{n}(\sigma)
$$

around $\sigma=0$, as can be readily proved from the integral expression for ${ }_{1} F_{1}\left(N ; N_{\mathrm{R}} ; \sigma\right)$ in [27. Eq. (13.4.1), p. 326].

Using (18), we rewrote (17) in [12, Eq. (37)] as the infinite series

$$
M_{\gamma_{1}}(s, a)=\sum_{n=0}^{\infty} A_{n}(a) \sum_{m=0}^{n}\left(\begin{array}{c}
n \\
m
\end{array}\right) \frac{(-1)^{m}}{\left(1-s \Gamma_{1}\right)^{N+n-m}} .
$$

Its inverse-Laplace transformation has yielded for the SNR p.d.f. the infinite series 2 [12, Eq. (39)]

$$
\begin{aligned}
p_{\gamma_{1}}(t, a)= & \sum_{n=0}^{\infty} A_{n}(a) \sum_{m=0}^{n}\left(\begin{array}{c}
n \\
m
\end{array}\right) \\
& \times \frac{(-1)^{m} t^{N+n-m-1} e^{-t / \Gamma_{1}}}{[(N+n-m)-1] ! \Gamma_{1}^{N+n-m}} .
\end{aligned}
$$

For the outage probability and ergodic capacity (in bits per channel use - bpcu), which are defined as 3

$$
\begin{gathered}
P_{\mathrm{o}}\left(\gamma_{1, \mathrm{th}}, a\right)=\operatorname{Probability}\left(\gamma_{1} \leq \gamma_{1, \mathrm{th}}\right)=\int_{0}^{\gamma_{1, \mathrm{th}}} p_{\gamma_{1}}(t, a) \mathrm{d} t, \\
C(a)=\mathbb{E}_{\gamma_{1}}\left\{C\left(\gamma_{1}, a\right)\right\}=\int_{0}^{\infty} \log _{2}(1+t) p_{\gamma_{1}}(t, a) \mathrm{d} t .
\end{gathered}
$$

analytical integration of the infinite-series p.d.f. expression (20) has yielded the infinite-series expressions in [12. Eqs. (69), (71)], respectively.

\footnotetext{
${ }^{2}$ Note that, for $K \neq 0$, i.e., for Rician-Rayleigh fading, 19 and 20 reveal that the distribution of the ZF SNR is an infinite linear combination of Gamma distributions.

${ }^{3}$ In $21, \gamma_{1, \text { th }}$ is the threshold SNR.
}

\section{Expressions for Rayleigh-Only Fading}

For the special case of Rayleigh-only fading, because $a=0$, only the term for $n=m=0$ remains in (19) and 20), i.e.,

$$
\begin{aligned}
M_{\gamma_{1}}(s, 0) & =\frac{1}{\left(1-s \Gamma_{1}\right)^{N}}, \\
p_{\gamma_{1}}(t, 0) & =\frac{t^{N-1} e^{-t / \Gamma_{1}}}{(N-1) ! \Gamma_{1}^{N}}, \quad t \geq 0,
\end{aligned}
$$

so that the ZF SNR is Gamma-distributed [7] [8]. Then, [21] and 22 yield

$$
\begin{aligned}
& P_{\mathrm{o}}\left(\gamma_{1, \mathrm{th}}, 0\right)=\frac{1}{(N-1) ! \Gamma_{1}^{N}} \int_{0}^{\gamma_{1, \mathrm{th}}} t^{N-1} e^{-t / \Gamma_{1}} \mathrm{~d} t \\
& C(0)=\frac{1}{\ln 2} \frac{1}{(N-1) !} \frac{1}{\Gamma_{1}^{N}} \int_{0}^{\infty} \ln (1+t) t^{N-1} e^{-t / \Gamma_{1}} \mathrm{~d} t .
\end{aligned}
$$

\section{Difficulties in Computing the Derived Infinite Series}

As mentioned in the Introduction, we proved analytically in [13] that the infinite series (20), along with the ensuing infinite series for the outage probability and ergodic capacity, converge everywhere. However, they cannot be computed (by truncation) accurately, or even at all, with increasing $K$ - see [12. Sections V.F, VI.C] [13] for discussion and results.

This limitation is also illustrated herein for the SNR p.d.f. infinite series in 20) in Fig. 1 for $N_{\mathrm{R}}=6, N_{\mathrm{T}}=2$. We have set $K=7 \mathrm{~dB}$ and $\mathrm{AS}=51^{\circ}$, i.e., the average $K$ and AS for WINNER II scenario A1 (indoor office/residential) [11. Table I] [14]. On one hand, results for Rayleigh-only fading (identified in legend with Ray-Ray) reveal agreement between expression (24) and Monte Carlo simulation. On the other hand, for Rician-Rayleigh fading (identified in legend with Rice-Ray), the results from series (20) are not usable. For $N_{\mathrm{R}}=6$ and $N_{\mathrm{T}}=2$, we have been able to accurately compute $p_{\gamma_{1}}(t)$, and, thus, the outage probability and ergodic capacity, only up to $K \approx 1.5 \mathrm{~dB}$, as depicted in [13, Fig. 2]. This is because, by increasing $K$, i.e., $a$, we move $\sigma$ further from the origin of expansion (18). Finally, we have also found that increasing $N_{\mathrm{T}}$ to 6 decreases the value of $K$ that still yields accurate results to $-3 \mathrm{~dB}$ [13, Figs. 1, 2], which is explained by (16).

Because infinite-series truncation cannot help compute MIMO ZF performance measures for relevant fading parameter values, we pursue next a novel HGM-based approach.

\section{Holonomic Functions AND the Holonomic GRADiEnT METHOD (HGM)}

\section{A. Differential Equation for ${ }_{1} F_{1}\left(N ; N_{R} ; \sigma\right)$}

It is known that ${ }_{1} F_{1}\left(N ; N_{\mathrm{R}} ; \sigma\right)$ satisfies the second-order ordinary differential equation with polynomial coefficients [27. Eq. (13.2.1), p. 322]

$$
\begin{aligned}
& \sigma \cdot{ }_{1} F_{1}^{(2)}\left(N ; N_{\mathrm{R}} ; \sigma\right)+\left(N_{\mathrm{R}}-\sigma\right) \cdot{ }_{1} F_{1}^{(1)}\left(N ; N_{\mathrm{R}} ; \sigma\right) \\
& -N \cdot{ }_{1} F_{1}\left(N ; N_{\mathrm{R}} ; \sigma\right)=0,
\end{aligned}
$$

\footnotetext{
${ }^{4}$ The finite- and infinite-limit integrals involved in these expressions can be computed accurately numerically.
} 


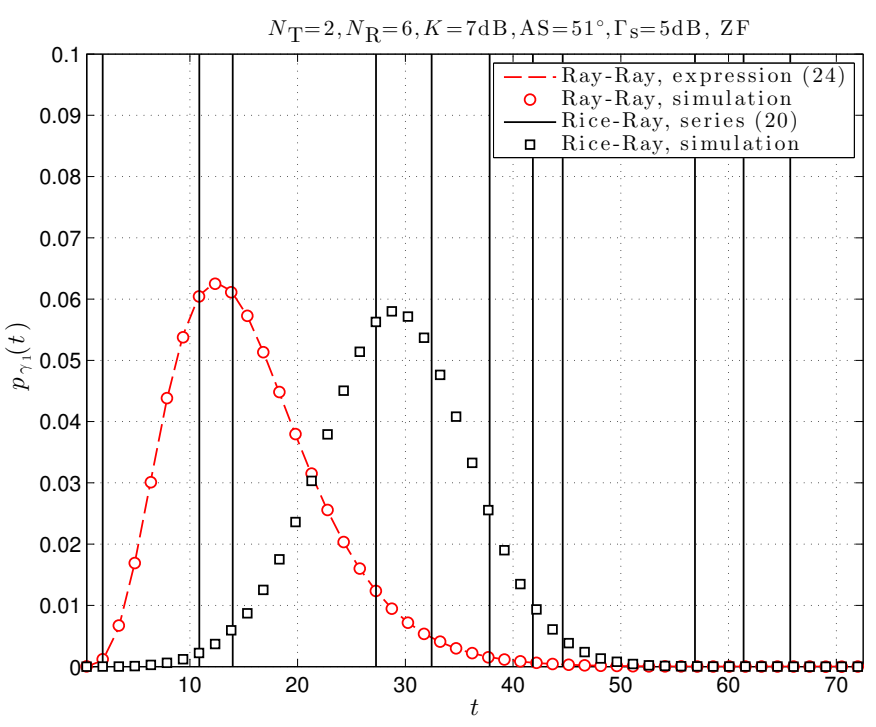

Fig. 1. P.d.f. of the SNR (in linear units) for Stream 1, for $N_{\mathrm{R}}=6, N_{\mathrm{T}}=2$, $K=7 \mathrm{~dB}, \mathrm{AS}=51^{\circ}, \Gamma_{\mathrm{s}}=5 \mathrm{~dB}$. For Rayleigh fading: from Monte Carlo simulation and expression 24. For Rician fading: from simulation and attempt to employ series 20; the latter produced the vertical black lines (which connect the points of extreme positive and negative values resulting from series truncation).

where ${ }_{1} F_{1}^{(k)}\left(N ; N_{\mathrm{R}} ; \sigma\right)$ stands for the $k$ th derivative w.r.t. the sole variable $\sigma$.

In general, a function is called holonomic if it satisfies, w.r.t. each variable, an ordinary differential equation with polynomial coefficients [37, Section 2]. Thus, the confluent hypergeometric function ${ }_{1} F_{1}\left(N ; N_{\mathrm{R}} ; \sigma\right)$ is holonomic because it satisfies (27). Simpler examples of holonomic functions are the polynomial and exponential-polynomial functions [33. Section 2.5].

Let us now introduce the HGM-based computation of a holonomic function on the example of ${ }_{1} F_{1}\left(N ; N_{\mathrm{R}} ; \sigma\right)$. First, 27) can be recast as the system of differential equations

$\partial_{\sigma}\left(\begin{array}{c}{ }_{1} F_{1}\left(N ; N_{\mathrm{R}} ; \sigma\right) \\ { }_{1} F_{1}^{(1)}\left(N ; N_{\mathrm{R}} ; \sigma\right)\end{array}\right)=\left(\begin{array}{cc}0 & 1 \\ \frac{N}{\sigma} & 1-\frac{N_{\mathrm{R}}}{\sigma}\end{array}\right)\left(\begin{array}{c}{ }_{1} F_{1}\left(N ; N_{\mathrm{R}} ; \sigma\right) \\ { }_{1} F_{1}^{(1)}\left(N ; N_{\mathrm{R}} ; \sigma\right)\end{array}\right)$.

If we denote as $\mathbf{f}(\sigma)$ the vector made of functions ${ }_{1} F_{1}\left(N ; N_{\mathrm{R}} ; \sigma\right)$ and ${ }_{1} F_{1}^{(1)}\left(N ; N_{\mathrm{R}} ; \sigma\right)$, and as $\mathbf{F}(\sigma)$ the $2 \times 2$ matrix on the right-hand side above, which is also known as the companion matrix, then, we have, more compactly,

$$
\partial_{\sigma} \mathbf{f}(\sigma)=\mathbf{F}(\sigma) \mathbf{f}(\sigma)
$$

whose left-hand side is the gradient of $\mathbf{f}(\sigma)$ w.r.t. $\sigma$.

\section{B. HGM-Based Computation of Holonomic Function}

Let us assume that initial conditions ${ }_{1} F_{1}\left(N ; N_{\mathrm{R}} ; \sigma_{0}\right)$ and ${ }_{1} F_{1}^{(1)}\left(N ; N_{\mathrm{R}} ; \sigma_{0}\right)$ are known for some $\sigma_{0}$. Then, ${ }_{1} F_{1}\left(N ; N_{\mathrm{R}} ; \sigma\right)$ can be computed for any $\sigma$ by numerically solving ${ }^{5}$ between $\sigma_{0}$ and $\sigma$. Because $\sigma$ appears in $\mathbf{F}(\sigma)$ denominators, one cannot use $\sigma_{0}=0$, for which it is known analytically, from [18) and [27. Eq. (13.3.15), p. 325],

\footnotetext{
${ }^{5}$ E.g., with the ode function, in MATLAB.
}

that ${ }_{1} F_{1}\left(N ; N_{\mathrm{R}} ; 0\right)=1$ and ${ }_{1} F_{1}^{(1)}\left(N ; N_{\mathrm{R}} ; 0\right)=\frac{N}{N_{\mathrm{R}}}$. Thus, initial conditions ${ }_{1} F_{1}\left(N ; N_{\mathrm{R}} ; \sigma_{0}\right)$ and ${ }_{1} F_{1}^{(1)}\left(N ; N_{\mathrm{R}} ; \sigma_{0}\right)=$ $\frac{N}{N_{\mathrm{R}}}{ }_{1} F_{1}\left(N+1 ; N_{\mathrm{R}}+1 ; \sigma_{0}\right)$ [27, Eq. (13.3.15), p. 325$]$ have to be obtained numerically by truncation of series $(18)$ for some $\sigma_{0}>0$. Nevertheless, since $\sigma_{0}$ can be selected arbitrarily small, highly-accurate computation of the initial condition $\mathbf{f}\left(\sigma_{0}\right)$ is possible based on the infinite-series expression of ${ }_{1} F_{1}\left(N ; N_{\mathrm{R}} ; \sigma\right)$ from $(18)$.

The entire procedure is summarized below [37, Section 2.1]:

- First, compute accurate initial conditions, i.e., ${ }_{1} F_{1}\left(N ; N_{\mathrm{R}} ; \sigma_{0}\right)$ and ${ }_{1} F_{1}^{(1)}\left(N ; N_{\mathrm{R}} ; \sigma_{0}\right)$, for a sufficientlysmall $\sigma_{0}>0$, by truncating infinite series (18).

- Then, solve numerically the system of differential equations (28) between $\sigma_{0}$ and $\sigma$.

This computation procedure (applicable to any holonomic function) is referred to as the holonomic gradient method [37] [38] (HGM) because, after starting from an initial condition, the holonomic function is computed by updating its gradient.

\section{ZF SNR M.G.F. and P.D.F. Are Holonomic Functions}

Holonomic functions satisfy the following properties:

1) If $f(x)$ is a polynomial then $1 / f(x)$ is holonomic $[33$ Prop. 2.1].

2) If $f(x)$ is holonomic and $h(x)$ is rational then $f(h(x))$ is holonomic [34, Th. 1.4.2, p. 16].

3) If $f(x)$ and $g(x)$ are holonomic then $f(x) g(x)$ is holonomic [33, Prop. 3.2].

4) If $f(x)$ is holonomic then its Fourier transform is holonomic [33, p. 337].

Properties 1 and 2 reveal that in the SNR m.g.f. expression (17), the term $1 /\left(1-\Gamma_{1} s\right)^{N}$ is holonomic w.r.t. $s$, and the term ${ }_{1} F_{1}\left(N ; N_{\mathrm{R}} ; a \frac{\Gamma_{1} s}{1-\Gamma_{1} s}\right)$ is holonomic w.r.t. $s$ and $a$. Then, Properties 3 and 4 prove the following lemma.

Lemma 1: The SNR m.g.f. $M_{\gamma_{1}}(s, a)$ is holonomic w.r.t. both $s$ and $a$, i.e., it must satisfy ordinary differential equations with polynomial coefficients w.r.t. both $s$ and $a$. Then, the SNR p.d.f. $p_{\gamma_{1}}(t, a)$ is holonomic w.r.t. both $t$ and $a$, i.e., it must satisfy ordinary differential equations with polynomial coefficients w.r.t. both $t$ and $a$.

\section{ZF Performance Analysis and Evaluation Procedure}

The rest of this work is devoted to deriving relevant differential equations and to using them for HGM-based $\mathrm{ZF}$ performance evaluation. The procedure is as follows:

1) Deduce differential equations - known to exist from Lemma 1 - satisfied by $M_{\gamma_{1}}(s, a)$ w.r.t. both $a$ and $s$, as well as by $p_{\gamma_{1}}(t, a)$ w.r.t. both $a$ and $t$. Considering both variables in each pair is necessary because, as we shall see, the partial derivatives w.r.t. the two variables appear together in differential equations for $M_{\gamma_{1}}(s, a)$ and for $p_{\gamma_{1}}(t, a)$.

2) Exploit the deduced differential equations for $p_{\gamma_{1}}(t, a)$ to compute the p.d.f. by HGM at relevant values of $K$.

3) Integrate numerically the p.d.f. produced by the HGM, based on (21), 22), to compute the outage probability and ergodic capacity. 


\section{Differential Equations For SNR M.G.F., P.D.F.}

\section{A. M.G.F. and P.D.F. Variable Scaling}

In order to simplify notation and derivations hereafter, let us denote the m.g.f. $M_{\gamma_{1}}(s, a)$ and the p.d.f. $p_{\gamma_{1}}(t, a)$ for $\Gamma_{1}=1$ as $M(s, a)$ and $p(t, a)$, respectively. Now, by definition, we have

$$
M(s, a)=\int_{0}^{\infty} e^{s t} p(t, a) \mathrm{d} t .
$$

Then, because

$$
\begin{aligned}
M_{\gamma_{1}}(s, a) & =M\left(s \Gamma_{1}, a\right)=\int_{0}^{\infty} e^{s \Gamma_{1} t} p(t, a) \mathrm{d} t \\
& =\int_{0}^{\infty} e^{s y} \frac{1}{\Gamma_{1}} p\left(\frac{y}{\Gamma_{1}}, a\right) \mathrm{d} y,
\end{aligned}
$$

the p.d.f. $p_{\gamma_{1}}(t, a)$ of $\gamma_{1}$ for any $\Gamma_{1}$ can be obtained from $p(t, a)$ as follows:

$$
p_{\gamma_{1}}(t, a)=\frac{1}{\Gamma_{1}} p\left(\frac{t}{\Gamma_{1}}, a\right) .
$$

Hereafter, we first derive differential equations for $M(s, a)$ w.r.t. both $s$ and $a$. From them we then deduce differential equations for $p(t, a)$ w.r.t. both $t$ and $a$. They will help compute, by HGM, the function $p(t, a)$ at desired values of $t$ and $a$ (i.e., $K$ ). Finally, the transformation from 30 will return the value of the SNR p.d.f. $p_{\gamma_{1}}(t, a)$, for any $\bar{\Gamma}_{1}$.

We proceed below as follows. First, we deduce the differential equation w.r.t. $s$ for $M(s, a)$. From it we then derive the differential equation w.r.t. $t$ for $p(t, a)$, which, in turn, helps derive the differential equation w.r.t. $a$ for $p(t, a)$.

\section{B. Differential Equation w.r.t. $s$ for $M(s, a)$}

Based on (17) and (30) we can write

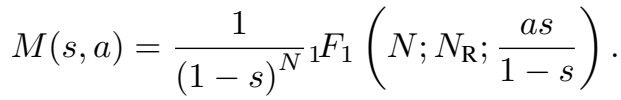

In Appendix A manipulation and differentiation w.r.t. $s$ of (31) followed by substitution into the differential equation for ${ }_{1} F_{1}\left(N ; N_{\mathrm{R}} ; \sigma\right)$ from 27 have yielded the following differential equation w.r.t. $s$ for $M(s, a)$, in 66):

$$
\begin{aligned}
\left(s(1-s)^{2} \partial_{s}^{2}-\right. & {\left[2(N+1) s(1-s)-(1-s) N_{\mathrm{R}}+a s\right] \partial_{s} } \\
+ & \left.N\left[(N+1) s-N_{\mathrm{R}}-a\right]\right) M(s, a)=0 .
\end{aligned}
$$

Because $s^{l}$ appears in front of $\partial_{s}^{k}$ in 32, the corresponding differential equation for $p(t, a)$ cannot be obtained by inverseLaplace transform. Therefore, we shall first employ the following order-changing rule, which can readily be deduced from [36. Th. 6.1.2 (Leibniz Formula), p. 282] [41, Th. 1.1.1, p. 3].

\section{Proposition 1:}

$s^{l} \partial_{s}^{k}=\sum_{m=0}^{\min (l, k)} \frac{(-1)^{m}(l-m+1)_{m}(k-m+1)_{m}}{m !} \partial_{s}^{k-m} s^{l-m}$.
The above general rule yields the following particular rules

$$
\begin{aligned}
s \partial_{s} & =\partial_{s} s-1, \\
s \partial_{s}^{2} & =\partial_{s}^{2} s-2 \partial_{s}, \\
s^{2} \partial_{s} & =\partial_{s} s^{2}-2 s, \\
s^{2} \partial_{s}^{2} & =\partial_{s}^{2} s^{2}-4 \partial_{s} s+2, \\
s^{3} \partial_{s}^{2} & =\partial_{s}^{2} s^{3}-6 \partial_{s} s^{2}+6 s,
\end{aligned}
$$

which, when applied in 32, yield for $M(s, a)$ the following differential equation w.r.t. $s$ :

$$
\begin{aligned}
& {\left[\partial_{s}^{2} s^{3}-2 \partial_{s}^{2} s^{2}+\partial_{s}^{2} s+(2 N-4) \partial_{s} s^{2}\right.} \\
& \quad+\left(6-2 N-N_{\mathrm{R}}-a\right) \partial_{s} s+\left(N_{\mathrm{R}}-2\right) \partial_{s} \\
& \quad+(N-1)(N-2) s \\
& \left.\quad+(N-1)\left(2-N_{\mathrm{R}}-a\right)\right] M(s, a)=0 .
\end{aligned}
$$

Unlike Eq. 32, Eq. 38 can be employed based on the inverse-Laplace transform to deduce the differential equation w.r.t. $t$ for $p(t, a)$, as shown next.

\section{Differential Equation w.r.t. $t$ for $p(t, a)$}

The following proposition helps transform an expression whereby the operator $\partial_{s}^{k}$ is applied to the product $s^{l} M(s, a)$ into a differential equation for $p(t, a)$ w.r.t. $t$. Hereafter, $p^{(l)}(t, a)$ stands for the $l$ th partial derivative w.r.t. $t$ of $p(t, a)$.

Proposition 2: The integral $\int_{0}^{\infty} e^{s t}\left[t^{k} p^{(l)}(t, a)\right] \mathrm{d} t$, which represents the Laplace transform of $t^{k} p^{(l)}(t, a)$ for argument $-s$, is given by:

$$
\left\{\begin{aligned}
(-1)^{l} \partial_{s}^{k}\left[s^{l} M(s, a)\right] & \\
+\sum_{m=k+1}^{l}(-1)^{m} p^{(l-m)}(0+, a) & \\
\times \frac{(m-1) !}{(m-k-1) !} s^{m-k-1}, & l \geq 1, \\
\partial_{s}^{k} M(s, a), & l=0 .
\end{aligned}\right.
$$

Proof: Follows from the well-known Laplace-transform property for higher-order derivatives from [27. Eq. (1.14.29), p. 28] and the sign change from (14).

Applying (39) for the terms in (38) yields the following Laplace-transform pairs:

$$
\begin{aligned}
& \partial_{s}^{2} s^{3} M(s, a)+2 ! p(0+, a) \leftrightarrow \\
& -t^{2} p^{(3)}(t, a) \\
& -2 \partial_{s}^{2} s^{2} M(s, a) \leftrightarrow \\
& -2 t^{2} p^{(2)}(t, a) \\
& \partial_{s}^{2} s M(s, a) \leftrightarrow \\
& -t^{2} p^{(1)}(t, a) \\
& (2 N-4) \partial_{s} s^{2} M(s, a)+(2 N-4) p(0+, a) \leftrightarrow \\
& (2 N-4) t p^{(2)}(t, a) \\
& \left(6-2 N-N_{\mathrm{R}}-a\right) \partial_{s} s M(s, a) \leftrightarrow \\
& -\left(6-2 N-N_{\mathrm{R}}-a\right) t p^{(1)}(t, a) \\
& \left(N_{\mathrm{R}}-2\right) \partial_{s} M(s, a) \leftrightarrow \\
& \left(N_{\mathrm{R}}-2\right) \operatorname{tp}(t, a) \\
& (N-1)(N-2) s M(s, a)+(N-1)(N-2) p(0+, a) \leftrightarrow \\
& -(N-1)(N-2) p^{(1)}(t, a) \\
& (N-1)\left(2-N_{\mathrm{R}}-a\right) M(s, a) \leftrightarrow \\
& (N-1)\left(2-N_{\mathrm{R}}-a\right) p(t, a) .
\end{aligned}
$$


Summing the left-hand-side terms (i.e., the $s$-domain terms) of the above transform pairs and accounting for 38 yield the constant $N(N-1) p(0+, a)$. However, this constant is zero because (30) above and (68) in Appendix B yield

$$
p(0+, a)= \begin{cases}{ }_{1} F_{1}\left(N ; N_{\mathrm{R}} ;-a\right), & N=1, \\ 0, & N>1 .\end{cases}
$$

Then, by the uniqueness of the Laplace transform, the right-hand-side terms (i.e., the $t$-domain terms) of the above transform pairs also sum to 0 , i.e.,

$$
\begin{aligned}
& -t^{2} p^{(3)}(t, a)-2 t^{2} p^{(2)}(t, a)-t^{2} p^{(1)}(t, a) \\
& \quad+(2 N-4) t p^{(2)}(t, a)-\left(6-2 N-N_{\mathrm{R}}-a\right) t p^{(1)}(t, a) \\
& \quad+\left(N_{\mathrm{R}}-2\right) t p(t, a)-(N-1)(N-2) p^{(1)}(t, a) \\
& \quad+(N-1)\left(2-N_{\mathrm{R}}-a\right) p(t, a)=0,
\end{aligned}
$$

which can be rewritten as the differential equation w.r.t. $t$

$$
\begin{aligned}
& p^{(3)}(t, a)=\frac{\left(N_{\mathrm{R}}-2\right) t+(N-1)\left(2-N_{\mathrm{R}}-a\right)}{t^{2}} p(t, a) \\
& -\frac{t^{2}+\left(6-2 N-N_{\mathrm{R}}-a\right) t+(N-1)(N-2)}{t^{2}} p^{(1)}(t, a) \\
& -\frac{2 t^{2}-(2 N-4) t}{t^{2}} p^{(2)}(t, a) .
\end{aligned}
$$

Finally, by defining the function vector

$$
\mathbf{p}(t, a)=\left(\begin{array}{lll}
p(t, a) & p^{(1)}(t, a) & p^{(2)}(t, a)
\end{array}\right)^{\mathcal{T}},
$$

we recast (42) as the system of differential equations w.r.t. $t$

$$
\partial_{t} \mathbf{p}(t, a)=\mathbf{P}(t, a) \mathbf{p}(t, a),
$$

where the elements of the $3 \times 3$ companion matrix $\mathbf{P}(t, a)$ are:

$$
\begin{aligned}
{[\mathbf{P}(t, a)]_{1,1}=} & {[\mathbf{P}(t, a)]_{1,3}=0, } \\
{[\mathbf{P}(t, a)]_{2,1}=} & {[\mathbf{P}(t, a)]_{2,2}=0, } \\
{[\mathbf{P}(t, a)]_{1,2}=} & {[\mathbf{P}(t, a)]_{2,3}=1, } \\
{[\mathbf{P}(t, a)]_{3,1}=} & \frac{\left(N_{\mathrm{R}}-2\right) t+(N-1)\left(2-N_{\mathrm{R}}-a\right)}{t^{2}}, \\
{[\mathbf{P}(t, a)]_{3,2}=} & -\frac{t^{2}+\left(6-2 N-N_{\mathrm{R}}-a\right) t}{t^{2}}, \\
{[\mathbf{P}(t, a)]_{3,3} } & =-\frac{2 t^{2}-(2 N-4) t}{t^{2}} .
\end{aligned}
$$

Note, however, that the system of differential equations w.r.t. $t$ in (44) was not deduced for directly solving the original problem. Thus, HGM w.r.t. $t$ based on (44) shall not be applied to compute the SNR p.d.f. for a given $a$ value (e.g., corresponding to a practical $K$ value) over a relevant SNR range starting at some $t_{0} \neq 0$. Such attempt would require $p\left(t_{0}, a\right), p^{(1)}\left(t_{0}, a\right)$, and $p^{(2)}\left(t_{0}, a\right)$ at the given $a$, but the infinite series available for them - shown in (20) and Appendix C - can be computed reliably only for small $a$, as explained in Section III-D

Nevertheless, the system of differential equations w.r.t. $t$ from (44) shall help indirectly in solving the original problem, because $\partial_{a} p^{(q)}(t, a)$ can be expressed in terms of $p^{(q)}(t, a)$, $q=0,1,2$, as shown next. The ensuing system of differential equations w.r.t. $a$ for $\mathbf{p}(t, a)$ may then be used for HGM-based computation of $p(t, a)$ at desired values of $a$.

\section{System of Differential Equations w.r.t. a for $\mathbf{p}(t, a)$}

In Appendix D, Eq. (79), we have deduced the relationship

$$
a \partial_{a} M(s, a)=\left(s \partial_{s}-s^{2} \partial_{s}-N s\right) M(s, a),
$$

which, by using rules 33 and 35 , becomes

$$
\begin{aligned}
a \partial_{a} M(s, a) & =\left(\partial_{s} s-1-\partial_{s} s^{2}+2 s-N s\right) M(s, a) \\
& =\left[-1+\left(-N s+\partial_{s} s+2 s\right)-\partial_{s} s^{2}\right] M(s, a) .
\end{aligned}
$$

Transformation of the above to the $t$-domain based on 39 . and further manipulation yield

$$
\begin{aligned}
a \partial_{a} p(t, a)= & \overbrace{(N-1) p(0+, a)}^{=0, \forall N}-p(t, a) \\
& +(N-t-2) p^{(1)}(t, a)-t p^{(2)}(t, a) \\
= & \mathbf{q}_{1}(t, a) \mathbf{p}(t, a),
\end{aligned}
$$

where $\mathbf{q}_{1}(t, a)=\left(\begin{array}{lll}-1 & N-t-2 \quad-t\end{array}\right)$.

Now, because $\partial_{a} p(t, a)$ depends on the entire vector $\mathbf{p}(t, a)$ defined in 43 , we have to also express $\partial_{a} p^{(1)}(t, a)$ and $\partial_{a} p^{(2)}(t, a)$ in terms of $\mathbf{p}(t, a)$, i.e., we have to express $\partial_{a} \mathbf{p}(t, a)$ in terms of $\mathbf{p}(t, a)$. This is achieved by differentiating 46 w.r.t. $t$ and substituting $p^{(3)}(t, a)$ from 42 twice, successively. The procedure yields the following expressions:

$$
\begin{aligned}
a \partial_{a} p^{(1)}(t, a) & \\
= & -t p^{(3)}(t, a)+(N-t-3) p^{(2)}(t, a)-2 p^{(1)}(t, a) \\
= & \left(2-N_{\mathrm{R}}+\frac{2-2 N-N_{\mathrm{R}}-a+N N_{\mathrm{R}}+N a}{t}\right) p(t, a) \\
& +\left(4-2 N-N_{\mathrm{R}}-a+t+\frac{2+N^{2}-3 N}{t}\right) p^{(1)}(t, a) \\
& +(1-N+t) p^{(2)}(t, a), \\
a \partial_{a} p^{(2)}(t, a) & \left(-2+N_{\mathrm{R}}+\frac{-4+4 N+2 N_{\mathrm{R}}+a-2 N N_{\mathrm{R}}-N a}{t}\right. \\
= & \left(-\frac{-4+6 N+2 N_{\mathrm{R}}+2 a-3 N N_{\mathrm{R}}-3 N a}{t^{2}}\right) p(t, a) \\
& +\frac{N^{2} N_{\mathrm{R}}+N^{2} a-2 N^{2}}{t^{2}} \\
+ & \left(3 N-4+a-t+\frac{-6-3 N^{2}+9 N}{t}\right. \\
& \left.+\frac{-4+8 N-5 N^{2}+N^{3}}{t^{2}}\right) p^{(1)}(t, a) \\
+ & \left(-1+2 N-N_{\mathrm{R}}-a-t\right. \\
& \left.+\frac{-2-N^{2}+3 N}{t}\right) p^{(2)}(t, a) .
\end{aligned}
$$

Finally, collecting (46)-48 yields for vector $\mathbf{p}(t, a)$ the system of differential equations w.r.t. $a$

$$
\partial_{a} \mathbf{p}(t, a)=\frac{1}{a} \mathbf{Q}(t, a) \mathbf{p}(t, a),
$$

where the first row of the $3 \times 3$ matrix $\mathbf{Q}(t, a)$ is $\mathbf{q}_{1}(t, a)$ and 
the remaining elements are as follows:

$$
\begin{aligned}
{[\mathbf{Q}(t, a)]_{2,1}=} & 2-N_{\mathrm{R}}+\frac{2-2 N-N_{\mathrm{R}}-a+N N_{\mathrm{R}}+N a}{t}, \\
{[\mathbf{Q}(t, a)]_{2,2}=} & 4-2 N-N_{\mathrm{R}}-a+t+\frac{2+N^{2}-3 N}{t}, \\
{[\mathbf{Q}(t, a)]_{2,3}=} & 1-N+t, \\
{[\mathbf{Q}(t, a)]_{3,1}=} & -2+N_{\mathrm{R}}+\frac{-4+4 N+2 N_{\mathrm{R}}}{t} \\
& +\frac{a-2 N N_{\mathrm{R}}-N a}{t} \\
& +\frac{-4+6 N+2 N_{\mathrm{R}}+2 a-3 N N_{\mathrm{R}}-3 N a}{t^{2}} \\
& +\frac{N^{2} N_{\mathrm{R}}+N^{2} a-2 N^{2}}{t^{2}}, \\
{[\mathbf{Q}(t, a)]_{3,2}=} & 3 N-4+a-t+\frac{-6-3 N^{2}+9 N}{t} \\
& +\frac{-4+8 N-5 N^{2}+N^{3}}{t^{2}}, \\
{[\mathbf{Q}(t, a)]_{3,3}=} & -1+2 N-N_{\mathrm{R}}-a-t+\frac{-2-N^{2}+3 N}{t} .
\end{aligned}
$$

Although the above derivations may appear to suggest that the system of differential equations w.r.t. $t$ from (44) is solely an instrument for deducing the one w.r.t. $a$ from (49), we explain next that (44) is as important as (49) in the HGMbased computation of the SNR p.d.f..

\section{E. Computation of $p(t, a)$ by $H G M$ w.r.t. a, Given $t$}

One may attempt to apply HGM w.r.t. $a$, i.e., to compute the SNR p.d.f. by solving (49) numerically between some small $a_{0}$ and the desired $a$ (i.e., $K$ ), given $t$ and initialcondition vector $\mathbf{p}\left(t, a_{0}\right)=\left(p\left(t, a_{0}\right) p^{(1)}\left(t, a_{0}\right) p^{(2)}\left(t, a_{0}\right)\right)^{\mathcal{T}}$ with elements computed by truncating their infinite series shown in 20 and in Appendix C.

However, we have found that, for moderate-to-large $t$, the value of $p\left(t, a_{0}\right)$ can be too small for sufficiently-accurate numerical representation, which prevents HGM w.r.t. $a$ from accurately computing $p(t, a)$ between $a_{0}$ and $a$.

Nevertheless, interestingly, we can avoid these numerical issues for HGM w.r.t. $a$ based on 49 by applying it in conjunction with HGM w.r.t. $t$ based on (44), as shown next.

\section{F. Computation of $p(t, a)$ by $H G M$, for $a=c t$}

In the systems of differential equations obtained in 44 and (49), i.e., in

$$
\begin{aligned}
\partial_{t} \mathbf{p}(t, a) & =\mathbf{P}(t, a) \mathbf{p}(t, a), \\
\partial_{a} \mathbf{p}(t, a) & =\frac{1}{a} \mathbf{Q}(t, a) \mathbf{p}(t, a),
\end{aligned}
$$

we now make the following changes of variables

$$
\begin{aligned}
t & =c_{1} u, \\
a & =c_{2} u .
\end{aligned}
$$

${ }^{6} \mathrm{HGM}$ requires $a_{0} \neq 0$ because $a$ divides matrix $\mathbf{Q}$ in 49 .
Then, the bivariate function vector from (43) becomes the univariate function vector

$$
\mathbf{p}\left(c_{1} u, c_{2} u\right)=\left(\begin{array}{c}
p\left(c_{1} u, c_{2} u\right) \\
p^{(1)}\left(c_{1} u, c_{2} u\right) \\
p^{(2)}\left(c_{1} u, c_{2} u\right)
\end{array}\right)=\widetilde{\mathbf{p}}(u) .
$$

Based on the chain rule [27, Eq. (1.5.7), p. 7] as well as on 50] and (51), we can write:

$$
\begin{aligned}
\frac{d}{d u} \widetilde{\mathbf{p}}(u) & =\frac{d}{d u} \mathbf{p}(\underbrace{c_{1} u}_{t}, \underbrace{c_{2} u}_{a}) \\
& =\left.\left[\partial_{t} \mathbf{p}(t, a) \frac{d t}{d u}+\partial_{a} \mathbf{p}(t, a) \frac{d a}{d u}\right]\right|_{\substack{t=c_{1} u \\
a=c_{2} u}} \\
& =c_{1} \mathbf{P}\left(c_{1} u, c_{2} u\right) \widetilde{\mathbf{p}}(u)+c_{2} \frac{1}{c_{2} u} \mathbf{Q}\left(c_{1} u, c_{2} u\right) \widetilde{\mathbf{p}}(u) \\
& =c_{1} \mathbf{P}\left(c_{1} u, c_{2} u\right) \widetilde{\mathbf{p}}(u)+\frac{1}{u} \mathbf{Q}\left(c_{1} u, c_{2} u\right) \widetilde{\mathbf{p}}(u) .(55)
\end{aligned}
$$

Then, for example, $c_{1}=1$ and $c_{2}=c$, i.e., $a=c t$, yields the system of differential equations

$$
\frac{d}{d u} \widetilde{\mathbf{p}}(u)=\left[\mathbf{P}(u, c u)+\frac{1}{u} \mathbf{Q}(u, c u)\right] \widetilde{\mathbf{p}}(u),
$$

which helps apply HGM for $a=c t$, i.e., simultaneously w.r.t. $a$ and $t$.

From $p(t, a)$ computed with the HGM as above we can recover the SNR p.d.f. $p_{\gamma_{1}}(t, a)$ with 30 . Finally, numerical integration of $p_{\gamma_{1}}(t, a)$ yields the outage probability and ergodic capacity based on 21) and (22, respectively.

\section{NumERICAL RESUlTS}

\section{A. Settings and Approach}

The numerical results presented below have been obtained for a MIMO system with $N_{\mathrm{R}}=6$ and $N_{\mathrm{T}}=2$ under RicianRayleigh fading with $K=7 \mathrm{~dB}$ and $\mathrm{AS}=51^{\circ}$, i.e., the means of their WINNER II lognormal distributions for the indoor scenario A1 [14], as follows:

- Monte-Carlo simulation: random samples of the channel matrix $\mathbf{H}$ have been generated based on the model (5); the ZF SNR for Stream 1 has been computed for each sample by using 12, a histogram of the samples has yielded the p.d.f. and c.d.f..

- Analysis: from expressions (24), 25 and (26) and from HGM for differential equation (56). When necessary, we have integrated numerically using the rectangle method.

We now outline our HGM-based procedure based on 56 for the computation of the ZF SNR p.d.f. and ensuing performance measures. Given $\Gamma_{\mathrm{s}}$ defined in (3) and the Rician $K$-factor, we have computed $\Gamma_{1}$ and $a$ with 15 and 16 , respectively. Then, we have computed the Stream-1 ZF SNR p.d.f. over the SNR range with the following steps:

1) Compute accurately the initial condition 7 $\widetilde{\mathbf{p}}\left(u_{0}\right)=$ $\left(p\left(u_{0}, u_{0}\right) p^{(1)}\left(u_{0}, u_{0}\right) p^{(2)}\left(u_{0}, u_{0}\right)\right)^{\mathcal{T}}$ for a sufficientlysmall $u_{0}$, using the infinite series for $p^{(q)}(t, a)$ derived in Appendix C.

${ }^{7}$ Note that both $t$ and $a$ are substituted with $u_{0}$ in this step, for simplicity. 


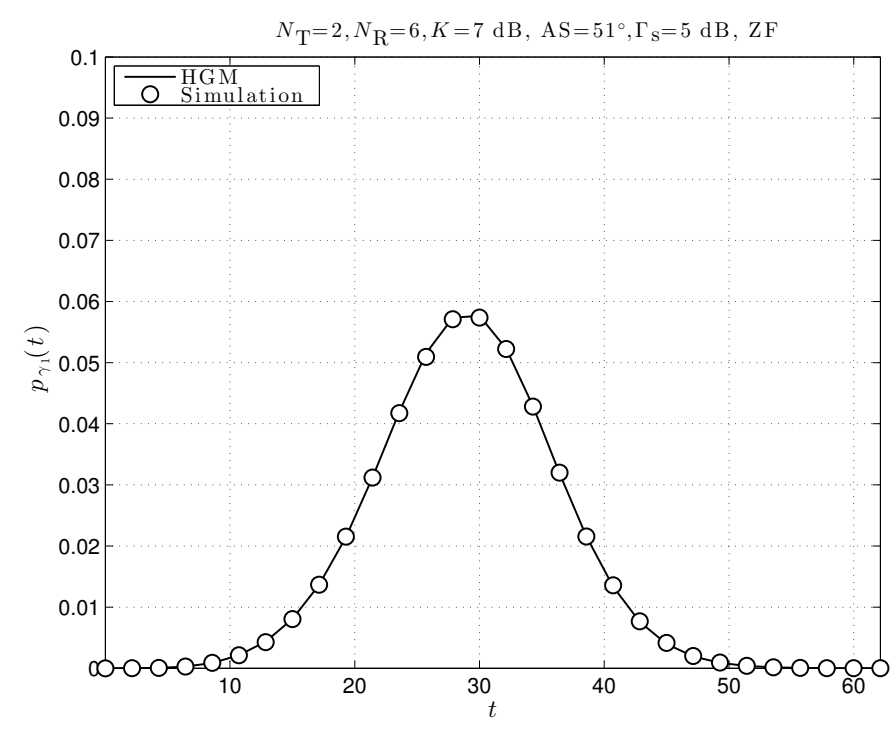

Fig. 2. Stream-1 SNR p.d.f. computed by the HGM, based on 56, and by Monte-Carlo simulation, for $N_{\mathrm{R}}=6, N_{\mathrm{T}}=2$, and Rician-Rayleigh fading with $K=7 \mathrm{~dB}, \mathrm{AS}=51^{\circ}$.

2) Sample the SNR range of interest $\left[u_{1}, u_{M}\right]$ as $u_{1}, u_{2}, \cdots, u_{M}$.

3) For each sample $u=u_{m}, m=1,2, \cdots, M$ : first, set $c=a / u$; then, apply HGM to solve 56 from $u_{0}$ to $u$; finally, save $p(u, c u)$, i.e., $p(t, a)$ on the line $a=c t$.

4) Recover the ZF SNR p.d.f. $p_{\gamma_{1}}(t, a)$ from $p(t, a)$ by 30 .

This approach avoids using the infinite series for $p(t, a)$, $p^{(1)}(t, a)$, and $p^{(2)}(t, a)$ at large $a$ or large $t$, and, thus, avoids the numerical issues described in Sections $\Pi I I-D$ and V-E.

Finally, we have computed the outage probability and ergodic capacity by numerically integrating, according to 21. and (22), respectively, the p.d.f. produced by HGM as described above.

\section{B. Description of Results}

Figs. 2 and 3 depict, respectively, the SNR p.d.f. and c.d.f. computed with the HGM as above, and by simulation. The HGM is successful, i.e., the resulting p.d.f. and c.d.f. agree with the simulation, and the c.d.f. shown in Fig 3 (from numerically integrating the p.d.f. produced by HGM) goes to 1 for increasing $t$. Recall that in [12] [13] we had been able to accurately compute $p_{\gamma_{1}}(t)$ based on its infinite series 20p only up to the unrealistically-small $K$ value of $1.5 \mathrm{~dB}$. This has also been illustrated herein in Fig. 1. where the seriesbased computation breaks down for $K=7 \mathrm{~dB}$. Consequently, Figs. 2 and 3 do not attempt to plot series results.

Finally, Figs. 4 and 5 depict, respectively, the outage probability and ergodic capacity (in bpcu) with respect to $\Gamma_{\mathrm{b}}$ defined in (4). For Rayleigh-only fading we have used the integral expressions (25) and (26), respectively. For RicianRayleigh fading we have integrated numerically according to (21) and 22), respectively, the SNR p.d.f. produced by HGM as shown above. The HGM and simulation results agree

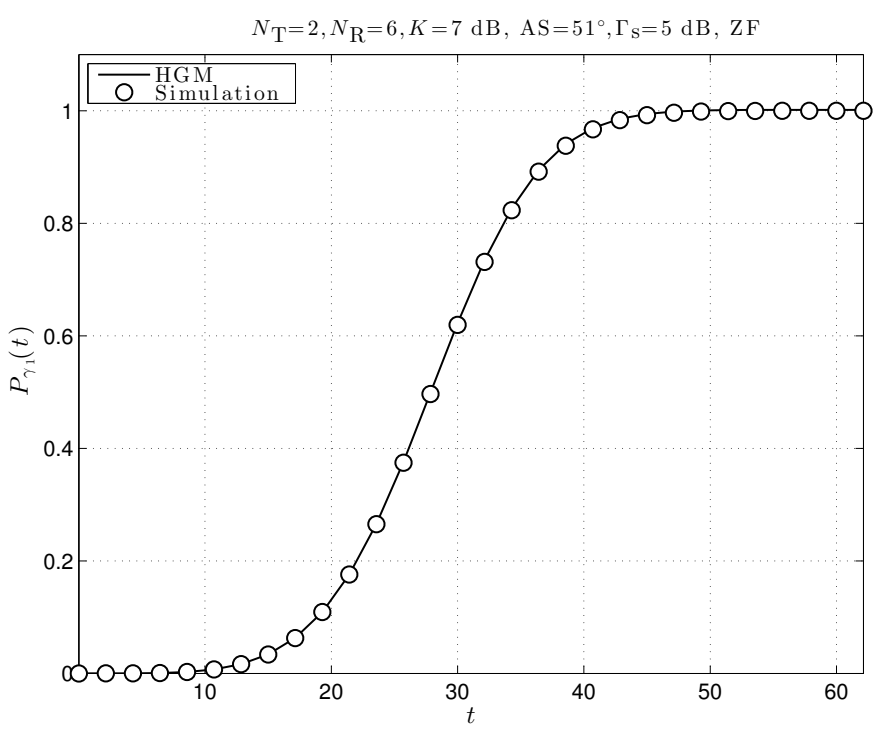

Fig. 3. Stream-1 SNR c.d.f. computed by numerical integration of the p.d.f. produced by HGM, based on 56, and by Monte-Carlo simulation, for $N_{\mathrm{R}}=6, N_{\mathrm{T}}=2$, and Rician-Rayleigh fading with $K=7 \mathrm{~dB}, \mathrm{AS}=51^{\circ}$.

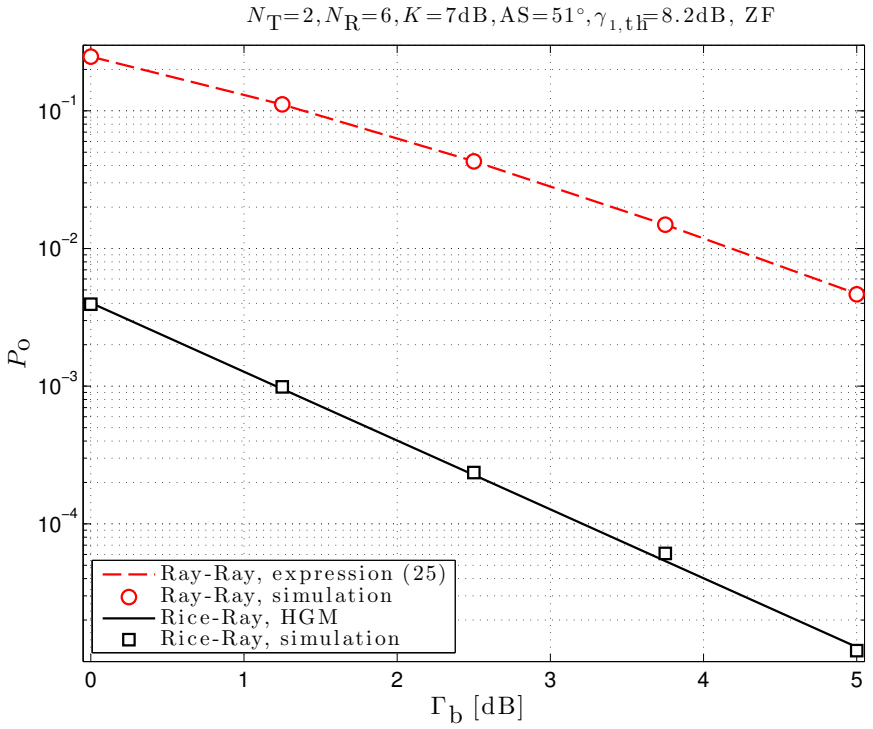

Fig. 4. Stream-1 outage probability for $N_{\mathrm{R}}=6, N_{\mathrm{T}}=2$, and fading parameters $K=7 \mathrm{~dB}, \mathrm{AS}=51^{\circ}$. For Rayleigh-only fading: from expression 25 and from simulation. For Rician-Rayleigh fading: from numerical integration according to 21] of the SNR p.d.f. computed with the HGM, based on 56, and from simulation.

closely 8 On the other hand, results from $P_{\mathrm{o}}$ and $C$ infinite series [12, Eqs. (69), (71)] could not be shown because their computation breaks down.

\section{HGM Complexity}

Our HGM-based computation solves (56) with the iterative Runge-Kutta method [27, Section 3.7] [37], for tolerance level $\epsilon=10^{-15}$ (i.e., 15 digits of accuracy), with the MATLAB

\footnotetext{
${ }^{8}$ Unshown results have revealed that HGM yields accurate results even for $K$ as high as $15 \mathrm{~dB}$, and also for other combinations of $N_{\mathrm{T}}$ and $N_{\mathrm{R}}$, without a noticeable increase in computation time.
} 


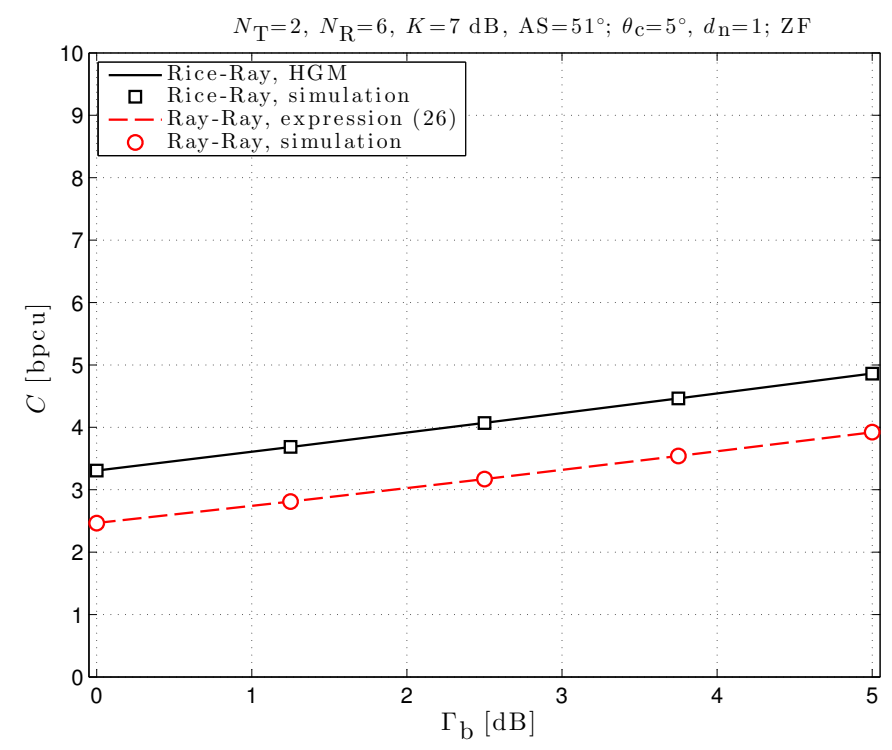

Fig. 5. Stream-1 ergodic capacity for $N_{\mathrm{R}}=6, N_{\mathrm{T}}=2$, and fading parameters $K=7 \mathrm{~dB}, \mathrm{AS}=51^{\circ}$. For Rayleigh-only fading: from expression 26 and from simulation. For Rician-Rayleigh fading: from numerical integration according to 22) of the SNR p.d.f. computed by HGM, based on 56, and from simulation.

ode function. Note that the Runge-Kutta method is available in most numerical tools, and its complexity is polynomial in the number of digits of accuracy [42]. Thus, we have found that the duration of the HGM-based computation is reasonable 9 Finally, HGM-based computation duration and success are robust to the value of $K$, unlike infinite-seriesbased computation [12] [13] [29].

\section{Future WORK: AN HGM-BASEd FrAMEWORK FOR MIMO EVALUATION}

\section{A. HGM-Based Evaluation of MIMO under General Fading}

For many fading types (e.g., Rayleigh, Rician, Nakagami, lognormal) and for many multiantenna transmission techniques, previous work, e.g. [2], showed expressions for the SNR m.g.f. that involve special (e.g., hypergeometric, Bessel) functions of scalar argument, which have typically been written as infinite-series, but are also holonomic. On the other hand, [32] analyzed MIMO performance under general fading by purposely writing as infinite series (ensuing from expansions around 0) the SNR m.g.f.. We have been applying a similar approach in [43] for $N_{\mathrm{R}} \times 2$ MIMO ZF under fullRician fading. The deduced SNR p.d.f. expression contains multiple infinite series whose computation by truncation has once again been found accurate only for unrealistically-small $K$ values. Therefore, we are currently attempting to apply instead the HGM-based approach: deduce and solve relevant differential equations.

Furthermore, hypergeometric functions also of matrix argument have often occurred in MIMO analyses due to sta-

\footnotetext{
${ }^{9}$ Computation of the p.d.f. at 30 samples of $t$, as shown in Fig. 2 requires about 60 seconds. Then, outage probability computation for one $T_{b}$ value takes about $70 \mathrm{~s}$. Finally, ergodic capacity computation for one $\Gamma_{\mathrm{b}}$ value takes about $140 \mathrm{~s}$
}

tistical assumptions about the channel matrix [30] [31]. For example, the c.d.f. and m.g.f. of the dominant eigenvalue of a complex-valued central-Wishart-distributed matrix have been expressed in terms of ${ }_{1} F_{1}(a ; c ; \mathbf{R})$ and ${ }_{2} F_{1}(a ; c ; \mathbf{R})$ in [31. Eqs. (34), (42)], respectively. Thus, for MIMO beamforming under Rayleigh-fading channel, the average error probability and outage probability have been expressed in terms of ${ }_{1} F_{1}(a ; c ; \mathbf{R})$ and ${ }_{2} F_{1}(a ; c ; \mathbf{R})$ in [31, Eqs. (30), (22)], respectively. Unfortunately, the well-known infinite series for these functions (and the zonal polynomials involved) [44. Eq. (1.1)] [30, Eq. (1.1)] [31, Eq. (61)] are difficult to compute [30] [31]. Nevertheless, such functions also satisfy differential equations [44] [45]. Those for ${ }_{1} F_{1}(a ; c ; \mathbf{R})$ from [44, Eq. (5.1)] have recently been exploited for the accurate HGM-based computation of the c.d.f. of the dominant eigenvalue of a real-valued central-Wishart-distributed matrix in [38]. We shall pursue this approach for complex-valued matrices, in order to evaluate MIMO beamforming performance.

\section{B. Computer-Algebra-Based Deduction of Equations}

In the current paper, HGM has been applied for solving differential equations for the ZF SNR m.g.f. and p.d.f. that we deduced manually. Computer-algebra tools that can help automate the deduction of differential equations for holonomic functions have recently become available [35, p. 171] [36. Ch. 7] [46] [47]. Thus, in other recent work [39], we have investigated employing and enhancing such tools to automatically derive differential equations not only for the ZF SNR m.g.f. and p.d.f., but also for the ZF outage probability and ergodic capacity. We shall continue investigating whether such automated tools can help apply HGM to MIMO performance evaluation under general fading, by deducing differential equations — instead of infinite series as in [2] [32] [43].

The outcome of this future work is envisioned to become a framework for the automated analysis and HGM-based evaluation of MIMO performance under general fading.

\section{SUMMARY AND CONCLUSIONS}

For MIMO ZF under Rician-Rayleigh fading, this paper has demonstrated that performance-measure expressions can be evaluated accurately, by using the HGM, at (realistic) Rician $K$-factor values that render unusable the conventional method of truncating infinite series. For the SNR m.g.f., which has been known in terms of the confluent hypergeometric function, which is a holonomic function, we have deduced the satisfied differential equations. They have yielded the differential equations satisfied by the SNR p.d.f., which, in turn, have helped compute the p.d.f. accurately using the HGM at values of $K$ relevant according to WINNER II (e.g., $K=7 \mathrm{~dB}$ ). Finally, numerical integration of the SNR p.d.f. obtained by the HGM has yielded for the MIMO ZF outage probability and ergodic capacity close agreement with simulations.

Future work shall attempt to extend the results of this paper into an automated HGM-based analysis and evaluation framework that promises to accurately characterize MIMO performance for realistic fading-parameter values. 


\section{APPENDIX A}

DIFFERENTIAL EQUATION W.R.T. $s$ FOR $M(s, a)$

First, substituting $\sigma$ with $\frac{a s}{1-s}$ in the differential equation for ${ }_{1} F_{1}\left(N ; N_{\mathrm{R}} ; \sigma\right)$ from 27 yields

$$
\begin{aligned}
& \frac{a s}{1-s}{ }_{1} F_{1}^{(2)}\left(N ; N_{\mathrm{R}} ; \frac{a s}{1-s}\right) \\
& \quad+\left(N_{\mathrm{R}}-\frac{a s}{1-s}\right){ }_{1} F_{1}^{(1)}\left(N ; N_{\mathrm{R}} ; \frac{a s}{1-s}\right) \\
& \quad-N_{1} F_{1}\left(N ; N_{\mathrm{R}} ; \frac{a s}{1-s}\right)=0 .
\end{aligned}
$$

Then, from 31, we have

$$
M(s, a)=\frac{1}{(1-s)^{N}}{ }_{1} F_{1}\left(N ; N_{\mathrm{R}} ; \frac{a s}{1-s}\right),
$$

which yields

$$
{ }_{1} F_{1}\left(N ; N_{\mathrm{R}} ; \frac{a s}{1-s}\right)=(1-s)^{N} M(s, a) .
$$

Differentiating (58) w.r.t. $s$ yields:

$$
\begin{aligned}
\partial_{s} M(s, a)= & \frac{N}{(1-s)^{N+1}{ }_{1} F_{1}\left(N ; N_{\mathrm{R}} ; \frac{a s}{1-s}\right)} \\
& +\frac{a}{(1-s)^{N+2}}{ }_{1} F_{1}^{(1)}\left(N ; N_{\mathrm{R}} ; \frac{a s}{1-s}\right)(60)
\end{aligned}
$$

By first substituting $[59]$ into $(60)$ and then by differentiating the result w.r.t. $s$ we obtain

$$
\begin{aligned}
\partial_{s} M(s, a)= & \frac{N}{(1-s)} M(s, a) \\
& +\frac{a}{(1-s)^{N+2}}{ }_{1} F_{1}^{(1)}\left(N ; N_{\mathrm{R}} ; \frac{a s}{1-s}\right)(61) \\
\partial_{s}^{2} M(s, a)= & \frac{N}{(1-s)^{2}} M(s, a)+\frac{N}{(1-s)} \partial_{s} M(s, a) \\
& +\frac{a(N+2)}{(1-s)^{N+3}}{ }_{1} F_{1}^{(1)}\left(N ; N_{\mathrm{R}} ; \frac{a s}{1-s}\right) \\
& +\frac{a^{2}}{(1-s)^{N+4}}{ }_{1} F_{1}^{(2)}\left(N ; N_{\mathrm{R}} ; \frac{a s}{1-s}\right)(62)
\end{aligned}
$$

which yield, respectively:

$$
\begin{aligned}
{ }_{1} F_{1}^{(1)} & \left(N ; N_{\mathrm{R}} ; \frac{a s}{1-s}\right) \\
= & \frac{(1-s)^{N+2}}{a}\left[\partial_{s}-\frac{N}{(1-s)}\right] M(s, a), \\
{ }_{1} F_{1}^{(2)}\left(N ; N_{\mathrm{R}} ; \frac{a s}{1-s}\right) & \\
= & \frac{(1-s)^{N+4}}{a^{2}}\left[\partial_{s}^{2} M(s, a)-\frac{N}{(1-s)^{2}} M(s, a)\right. \\
& -\frac{N}{(1-s)} \partial_{s} M(s, a) \\
& \left.-\frac{a(N+2)}{(1-s)^{N+3}}{ }_{1} F_{1}^{(1)}\left(N ; N_{\mathrm{R}} ; \frac{a s}{1-s}\right)\right] .
\end{aligned}
$$

Substituting 63) into 64) yields:

$$
\begin{aligned}
{ }_{1} F_{1}^{(2)}\left(N ; N_{\mathrm{R}} ; \frac{a s}{1-s}\right)= & \frac{(1-s)^{N+4}}{a^{2}}\left[\partial_{s}^{2}-\frac{2(N+1)}{(1-s)} \partial_{s}\right. \\
& \left.+\frac{N(N+1)}{(1-s)^{2}}\right] M(s, a) . \quad \text { (65) }
\end{aligned}
$$

Finally, substituting $(59), 63)$, and 655 into the differential equation (57), and further manipulation, yield the following differential equation w.r.t. $s$ for $M(s, a)$

$$
\begin{aligned}
& \left(s(1-s)^{2} \partial_{s}^{2}-\left[2(N+1) s(1-s)-(1-s) N_{\mathrm{R}}+a s\right] \partial_{s}\right. \\
& \left.\quad+N\left[(N+1) s-N_{\mathrm{R}}-a\right]\right) M(s, a)=0,
\end{aligned}
$$

which appears in the main text in (32).

\section{APPENDIX B}

INITIAL CONDITION $p_{\gamma_{1}}(0+, a)$

For the special case with $N=1$, i.e., for $N_{\mathrm{R}}=N_{\mathrm{T}}$, 20, becomes

$p_{\gamma_{1}}(t, a)=\frac{e^{-t / \Gamma_{1}}}{\Gamma_{1}} \sum_{n=0}^{\infty} A_{n}(a) \sum_{m=0}^{n}\left(\begin{array}{c}n \\ m\end{array}\right) \frac{(-1)^{m} t^{n-m}}{(n-m) ! \Gamma_{1}^{n-m}}$,

which yields

$$
\begin{aligned}
\lim _{t \rightarrow 0, t>0} p_{\gamma_{1}}(t, a) & =p_{\gamma_{1}}(0+, a)=\frac{1}{\Gamma_{1}} \sum_{n=0}^{\infty} A_{n}(a)(-1)^{n} \\
& =\frac{1}{\Gamma_{1}} \sum_{n=0}^{\infty} \frac{(N)_{n}}{\left(N_{\mathrm{R}}\right)_{n}} \frac{(-a)^{n}}{n !}
\end{aligned}
$$

Thus, (67), (18), and 20) yield

$$
p_{\gamma_{1}}(0+, a)= \begin{cases}\frac{1}{\Gamma_{1}} 1 F_{1}\left(N ; N_{\mathrm{R}} ;-a\right), & N=1, \\ 0, & N>1,\end{cases}
$$

which is used in the main text to deduce (40).

\section{APPENDIX C}

INFINITE SERIES FOR DERIVATIVES OF $p(t, a)$ W.R.T. $t$

Based on (20) and (30), let us define the function

$$
\begin{aligned}
f(t, a)=p(t, a) e^{t}= & \sum_{n=0}^{\infty} A_{n}(a) \sum_{m=0}^{n}\left(\begin{array}{c}
n \\
m
\end{array}\right) \\
& \times \frac{(-1)^{m} t^{N+n-m-1}}{(N+n-m-1) !},
\end{aligned}
$$

whose first two derivatives are given by

$$
\begin{aligned}
& f^{(1)}(t, a)=p^{(1)}(t, a) e^{t}+p(t, a) e^{t}, \\
& f^{(2)}(t, a)=p^{(2)}(t, a) e^{t}+2 p^{(1)}(t, a) e^{t}+p(t, a) e^{t} .
\end{aligned}
$$

The above yield

$$
p(t, a)=f(t, a) e^{-t},
$$

as well as

$$
\begin{aligned}
& p^{(1)}(t, a)=\left[f^{(1)}(t, a)-f(t, a)\right] e^{-t}, \\
& p^{(2)}(t, a)=\left[f^{(2)}(t, a)-2 f^{(1)}(t, a)+f(t, a)\right] e^{-t}(72)
\end{aligned}
$$


TABLE I

DeRIVATIVES OF $f(t, a)$ FOR $N=1,2$

\begin{tabular}{c|c|c}
\hline & $N=1$ & $N=2$ \\
\hline$f(t, a)$ & $g(t, a)$ & $t g(t, a)$ \\
\hline$f^{(1)}(t, a)$ & $g^{(1)}(t, a)$ & $g(t, a)+t g^{(1)}(t, a)$ \\
\hline$f^{(2)}(t, a)$ & $g^{(2)}(t, a)$ & $2 g^{(1)}(t, a)+t g^{(2)}(t, a)$ \\
\hline
\end{tabular}

which are the only derivatives of $p(t, a)$ required for 43 .

Now, if we rewrite $f(t, a)$ from 69 further as

$$
\begin{aligned}
f(t, a) & =t^{N-1} \underbrace{\sum_{n=0}^{\infty} A_{n}(a) \sum_{m=0}^{n}\left(\begin{array}{c}
n \\
m
\end{array}\right) \frac{(-1)^{m} t^{n-m}}{(N-1+n-m) !}}_{g(t, a)} \\
& =t^{N-1} g(t, a),
\end{aligned}
$$

then its $q$ th partial derivative w.r.t. $t$ is 10 based on Leibniz's formula [27, Eq. (1.4.12), p. 5]:

$$
\begin{aligned}
f^{(q)}(t, a) & =\partial_{t}^{q}\left[t^{N-1} g(t, a)\right] \\
& =\sum_{k=0}^{q}\left(\begin{array}{l}
q \\
k
\end{array}\right)\left[t^{N-1}\right]^{(k)} g^{(q-k)}(t, a) \\
& =\sum_{k=0}^{q}\left(\begin{array}{l}
q \\
k
\end{array}\right) \frac{(N-1) ! t^{N-1-k} g^{(q-k)}(t, a)}{(N-1-k) !}
\end{aligned}
$$

If we rewrite $g(t, a)$ from (73) as

$$
g(t, a)=\sum_{n=0}^{\infty} A_{n}(a) \sum_{r=0}^{n}\left(\begin{array}{c}
n \\
n-r
\end{array}\right) \frac{(-1)^{n-r} t^{r}}{(N-1+r) !}
$$

then its partial derivative of order $q \geq 1$ w.r.t. $t$ is given by

$$
\begin{aligned}
g^{(q)}(t, a)= & \sum_{n=q}^{\infty} A_{n}(a) \sum_{r=q}^{n}\left(\begin{array}{c}
n \\
n-r
\end{array}\right) \\
& \times \frac{(-1)^{n-r}}{(N-1+r) !} \frac{r !}{(r-q) !} t^{r-q},
\end{aligned}
$$

which, along with 75 , yields $f^{(q)}(t, a)$. Finally, substituting into 710 and 72 yields expressions for $p^{(1)}(t, a)$ and $p^{(2)}(t, a)$, respectively.

However, because (75) follows from (74) only for $k \leq N-$ 1 , and because $k$ goes from 0 to $q$, it is required that $N-1 \geq$ $q$. Then, because 43 requires $f^{(q)}(t, a)$ for $q$ as high as 2 , $f^{(q)}(t, a)$ can be written as in 75 only if $N \geq 3$. Table I characterizes the remaining cases.

\section{APPENDIX D \\ RELATIONSHIP BETWEEN DERIVATIVES of $M(s, a)$ W.R.T. $s$ AND $a$}

Differentiating 580 w.r.t. $a$ yields

$$
\partial_{a} M(s, a)=\frac{s}{(1-s)^{N+1}}{ }_{1} F_{1}^{(1)}\left(N ; N_{\mathrm{R}} ; \frac{a s}{1-s}\right),
$$

so that

$$
{ }_{1} F_{1}^{(1)}\left(N ; N_{\mathrm{R}} ; \frac{a s}{1-s}\right)=\frac{(1-s)^{N+1}}{s} \partial_{a} M(s, a) .
$$

${ }^{10}$ Note that 75 follows from 74 only for $k \leq N-1$.
Now, by substituting (59) and (78) into (60), and by further manipulation, we obtain

$$
a \partial_{a} M(s, a)=s(1-s) \partial_{s} M(s, a)-N s M(s, a),
$$

which appears in the main text in (45).

\section{ACKNOWLEDGMENTS}

The first version of this paper was prepared when the first author was with the Graduate School of Information Science and Technology, University of Tokyo, supported by Japan Science and Technology Agency. These institutions also supported the publication of this paper. The second (accepted) version was prepared after he joined the Graduate School of Information Science and Technology, Osaka University.

Akimichi Takemura acknowledges the support of the Japan Society for the Promotion of Science (JSPS) grant-in-aid for scientific research No. 25220001 and the support of Japan Science and Technology Agency.

Hyundong Shin acknowledges the support of the National Research Foundation of Korea (NRF) grants No. 20090083495 and No. 2013-R1A1A2-019963, funded by the Ministry of Science, ICT \& Future Planning (MSIP).

Christoph Koutschan acknowledges the support of the Austrian Science Fund (FWF): W1214.

\section{REFERENCES}

[1] A. J. Paulraj, D. A. Gore, R. U. Nabar, and H. Bolcskei, "An overview of MIMO communications-A key to gigabit wireless," Proc. of the IEEE, vol. 92, no. 2, pp. 198-218, February 2004.

[2] M. K. Simon and M.-S. Alouini, Digital Communication Over Fading Channels. A Unified Approach to Performance Analysis. Baltimore, Maryland: John Wiley and Sons, 2000.

[3] D. Gesbert, M. Kountouris, R. W. Heath, C.-B. Chae, and T. Salzer, "Shifting the MIMO paradigm," IEEE Signal Processing Magazine, vol. 24, no. 5, pp. 36-46, 2007.

[4] M. McKay, A. Zanella, I. Collings, and M. Chiani, "Error probability and SINR analysis of optimum combining in Rician fading," IEEE Transactions on Communications, vol. 57, no. 3, pp. 676-687, March 2009.

[5] J. Hoydis, S. ten Brink, and M. Debbah, "Massive MIMO in the UL/DL of cellular networks: How many antennas do we need?" IEEE Journal on Selected Areas in Communications, vol. 31, no. 2, pp. 160-171, 2013.

[6] R. Louie, M. McKay, and I. Collings, "New performance results for multiuser optimum combining in the presence of Rician fading," IEEE Transactions on Communications, vol. 57, no. 8, pp. 2348-2358, Aug. 2009.

[7] D. A. Gore, R. W. Heath Jr, and A. J. Paulraj, "Transmit selection in spatial multiplexing systems," IEEE Communications Letters, vol. 6, no. 11, pp. 491-493, 2002.

[8] M. Kiessling and J. Speidel, "Analytical performance of MIMO zeroforcing receivers in correlated Rayleigh fading environments," in IEEE Workshop on Signal Processing Advances in Wireless Communications (SPAWC'03), June 2003, pp. 383-387.

[9] M. Matthaiou, C. Zhong, M. McKay, and T. Ratnarajah, "Sum rate analysis of ZF receivers in distributed MIMO systems," IEEE Journal on Selected Areas in Communications, vol. 31, no. 2, pp. 180-191, 2013.

[10] D. A. Basnayaka, P. J. Smith, and P. A. Martin, "Performance analysis of macrodiversity MIMO systems with MMSE and ZF receivers in flat Rayleigh fading," IEEE Trans. on Wireless Communications, vol. 12, no. 5, pp. 2240-2251, 2013.

[11] C. Siriteanu, Y. Miyanaga, S. D. Blostein, S. Kuriki, and X. Shi, "MIMO zero-forcing detection analysis for correlated and estimated Rician fading," IEEE Transactions on Vehicular Technology, vol. 61, no. 7, pp. 3087-3099, September 2012.

[12] C. Siriteanu, S. D. Blostein, A. Takemura, H. Shin, S. Yousefi, and S. Kuriki, "Exact MIMO zero-forcing detection analysis for transmitcorrelated Rician fading," IEEE Transactions on Wireless Communications, vol. 13, no. 3, pp. 1514-1527, March 2014. 
[13] C. Siriteanu, A. Takemura, S. D. Blostein, S. Kuriki, and H. Shin, "Convergence analysis of performance-measure expressions for MIMO ZF under Rician fading," in Australian Communications Theory Workshop, (AUSCTW'14), Sydney, Australia, Feb. 2014

[14] P. Kyosti, J. Meinila, L. Hentila, and et al., "WINNER II Channel Models. Part I," CEC, Tech. Rep. IST-4-027756, 2008.

[15] L. Bai and J. Choi, Low complexity MIMO detection. Springer, 2012.

[16] Q. Li, G. Li, W. Lee, M. Lee, D. Mazzarese, B. Clerckx, and Z. Li, "MIMO techniques in WiMAX and LTE: a feature overview," IEEE Communications Magazine, vol. 48, no. 5, pp. 86-92, May 2010.

[17] M. Jung, Y. Kim, J. Lee, and S. Choi, "Optimal number of users in zeroforcing based multiuser MIMO systems with large number of antennas," Journal of Communications and Networks, vol. 15, no. 4, pp. 362-369, 2013.

[18] H. Ngo, E. Larsson, and T. Marzetta, "The multicell multiuser MIMO uplink with very large antenna arrays and a finite-dimensional channel," IEEE Transactions on Communications, vol. 61, no. 6, pp. 2350-2361, 2013.

[19] H. Artes, D. Seethaler, and F. Hlawatsch, "Efficient detection algorithms for MIMO channels: a geometrical approach to approximate ML detection," IEEE Transactions on Signal Processing, vol. 51, no. 11, pp. 2808-2820, Nov. 2003.

[20] D. Gesbert, "Robust linear MIMO receivers: A minimum error-rate approach," IEEE Transactions on Signal Processing, vol. 51, no. 11, pp. 2863-2871, 2003

[21] X. Ma and W. Zhang, "Fundamental limits of linear equalizers: diversity, capacity, and complexity," IEEE Transactions on Information Theory, vol. 54, no. 8, pp. 3442-3456, 2008.

[22] J. Maurer, J. Jaldén, D. Seethaler, and G. Matz, "Achieving a continuous diversity-complexity tradeoff in wireless MIMO systems via preequalized sphere-decoding," IEEE Journal of Selected Topics in Signal Processing, vol. 3, no. 6, pp. 986-999, 2009.

[23] J. H. Winters, J. Salz, and R. D. Gitlin, "The impact of antenna diversity on the capacity of wireless communication systems," IEEE Transactions on Communications, vol. 42, no. 234, pp. 1740-1751, 1994.

[24] P. Li, D. Paul, R. Narasimhan, and J. Cioffi, "On the distribution of SINR for the MMSE MIMO receiver and performance analysis," IEEE Transactions on Information Theory, vol. 52, no. 1, pp. 271-286, 2006

[25] Y. Jiang, M. Varanasi, and J. Li, "Performance analysis of ZF and MMSE equalizers for MIMO systems: An in-depth study of the high SNR regime," IEEE Transactions on Information Theory, vol. 57, no. 4 pp. 2008-2026, April 2011.

[26] A. H. Mehana and A. Nosratinia, "Diversity of MMSE MIMO receivers," IEEE Transactions on Information Theory, vol. 58, no. 11, pp. 6788-6805, 2012.

[27] F. W. J. Olver, D. W. Lozier, R. F. Boisvert, and C. W. Clarck, Eds. NIST Handbook of Mathematical Functions. Cambridge University Press, 2010.

[28] K. J. Kim, Y. Fan, R. A. Iltis, H. V. Poor, and M. H. Lee, "A reduced feedback precoder for MIMO-OFDM cooperative diversity systems," IEEE Transactions on Vehicular Technology, vol. 61, no. 2, pp. 584596, Feb. 2012

[29] K. E. Muller, "Computing the confluent hypergeometric function, $M(a, b, x)$, , Numerische Mathematik, vol. 90, no. 1, pp. 179-196, 2001

[30] P. Koev and A. Edelman, "The efficient evaluation of the hypergeometric function of a matrix argument," Mathematics of Computation, vol. 75, no. 254, pp. 833-846, 2006.

[31] A. J. Grant, "Performance analysis of transmit beamforming," IEEE Transactions on Communications, vol. 53, no. 4, pp. 738-744, 2005.

[32] G. A. Ropokis, A. A. Rontogiannis, P. T. Mathiopoulos, and K. Berberidis, "An exact performance analysis of MRC/OSTBC over generalized fading channels," IEEE Transactions on Communications, vol. 58 , no. 9, pp. 2486-2492, 2010

[33] D. Zeilberger, "A holonomic systems approach to special functions identities," Journal of computational and applied mathematics, vol. 32, no. 3, pp. 321-368, 1990

[34] C. Mallinger, "Algorithmic manipulations and transformations of univariate holonomic functions and sequences," Ph.D. dissertation, Johannes Kepler University Linz, Austria, 1996.

[35] M. Kauers and P. Paule, The Concrete Tetrahedron: Symbolic Sums, Recurrence Equations, Generating Functions, Asymptotic Estimates. Springer Wien, 2011

[36] T. Hibi, Ed., Grobner Bases. Statistics and Software Systems. Tokyo, Japan: Springer, 2013.

[37] T. Sei and A. Kume, "Calculating the normalising constant of the Bingham distribution on the sphere using the holonomic gradient method," Statistics and Computing, pp. 1-12, 2013.
[38] H. Hashiguchi, Y. Numata, N. Takayama, and A. Takemura, "The holonomic gradient method for the distribution function of the largest root of a Wishart matrix," Journal of Multivariate Analysis, vol. 117, pp. 296-312, 2013.

[39] C. Siriteanu, C. Koutschan, T. Onoye, and A. Takemura, "Computeralgebra based MIMO zero-forcing detection performance analysis," in IEEE Int. Conf. on Comm., (ICC'15), London, UK, 2015, submitted.

[40] S. Loyka and G. Levin, "On physically-based normalization of MIMO channel matrices," IEEE Transactions on Wireless Communications, vol. 8, no. 3, pp. 1107-1112, March 2009.

[41] M. Saito, B. Sturmfels, and N. Takayama, Gröbner Deformations of Hypergeometric Differential Equations, ser. Algorithms and Computation in Mathematics. Springer, 2011

[42] S. Ilie, G. Söderlind, and R. M. Corless, "Adaptivity and computational complexity in the numerical solution of ODEs," Journal of Complexity, vol. 24 , no. 3, pp. 341-361, 2008.

[43] C. Siriteanu, A. Takemura, S. Kuriki, D. Richards, T. Onoye, and H. Shin, "Performance analysis of $N_{\mathrm{R}} \times 2$ MIMO zero-forcing for fullRician fading," IEEE Transactions on Wireless Communications, to be submitted, 2015

[44] R. J. Muirhead, "Systems of partial differential equations for hypergeometric functions of matrix argument," The Annals of Mathematical Statistics, vol. 41, no. 3, pp. 991-1001, 1970.

[45] Y. Chikuse, "Partial differential equations for hypergeometric functions of complex argument matrices and their applications," Annals of the Institute of Statistical Mathematics, vol. 28, no. 1, pp. 187-199, 1976.

[46] C. Koutschan, "Advanced applications of the holonomic systems approach," Ph.D. dissertation, Research Institute for Symbolic Computation (RISC), Johannes Kepler University, Linz, Austria, 2009. [Online]. Available: http://www.risc.jku.at/research/combinat/ software/HolonomicFunctions/

[47] —- "HolonomicFunctions (user's guide)," RISC Report Series, Johannes Kepler University, Linz, Austria, Tech. Rep. 10-01, 2010. [Online]. Available: http://www.risc.jku.at/research/combinat/software/ HolonomicFunctions/

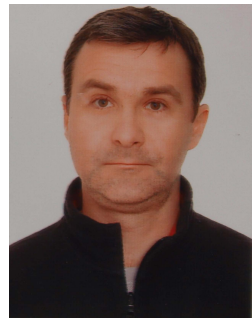

Constantin (Costi) Siriteanu was born in Sibiu, Romania. He received the Bachelor and Master degrees in Control Systems from "Gheorghe Asachi" Technical University, Iasi, Romania, in 1995 and 1996, respectively, and the Ph.D. degree in Electrical and Computer Engineering from Queen's University, Canada, in 2006. His Ph.D. thesis was on the performance-complexity tradeoff for smart antennas. Between September 2006 and March 2014 he worked as Researcher and Assistant Professor in Korea (Seoul National University, Kyung Hee Univesity, Hanyang University), Canada (Queen's University), and Japan (Hokkaido University, University of Tokyo). Since April 2014, he is a CAREN Specially-Appointed Assistant Professor with the Graduate School of Information Science and Technology, Osaka University. His research interests have been in developing multivariate statistics concepts that help analyze and evaluate the performance of multiple-input/multiple-output (MIMO) wireless communications systems under realistic statistical assumptions about channel fading. Recently, Constantin has been working on applications of computer algebra to the deduction of implicit representations of MIMO performance measures (i.e., as solutions of differential equations). 


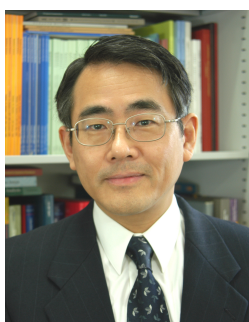

Akimichi Takemura received the Bachelor of Arts degree in Economics in 1976 and the Master of Arts degree in Statistics in 1978 from University of Tokyo, and the Ph.D. degree in Statistics in 1982 from Stanford University. He was an acting Assistant Professor at the Department of Statistics, Stanford University from September 1992 to June 1983, and a visiting Assistant Professor at the Department of Statistics, Purdue University from September 1983 to May 1984. In June 1984 he has joined University of Tokyo, where he has been a Professor of Statistics with the Department of Mathematical Informatics since April 2001. He has served as President of Japan Statistical Society from January 2011 to June 2013. He has been working on multivariate distribution theory in statistics. Currently his main area of research is algebraic statistics. He also works on game-theoretic probability, which is a new approach to probability theory.

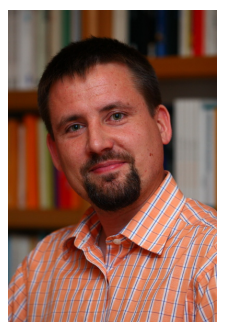

Christoph Koutschan received the Master degree in Computer Science from Friedrich-Alexander University in Erlangen, Germany, and the Ph.D. degree in Symbolic Computation from the Johannes Kepler University in Linz, Austria. He worked as a researcher at the Research Institute for Symbolic Computation (RISC, Linz, Austria), at Tulane University (New Orleans, USA), and at INRIA (Institut national de recherche en informatique et en automatique, France). Currently he is with the Johann Radon Institute for Computational and Applied Mathematics (RICAM) of the Austrian Academy of Sciences. His research interests are on methods related to the holonomic systems approach, particularly symbolic summation and integration algorithms, and their application to problems from combinatorics, knot theory, special functions, numerical analysis, and statistical physics.

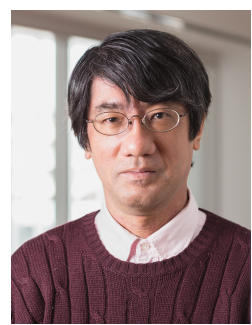

Satoshi Kuriki received the Bachelor and Ph.D. degrees from University of Tokyo, Japan, in 1982 and 1993, respectively. He is a Professor with the Institute of Statistical Mathematics (ISM), Tokyo, Japan, where he is also serving as Director of the Department of Mathematical Analysis and Statistical Inference. His current major research interests include geometry of random fields, multivariate analysis, multiple comparisons, graphical models, optimal designs, and genetic statistics.

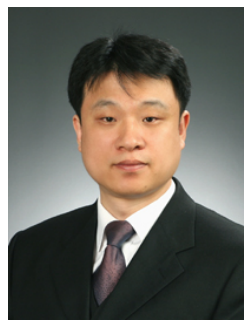

Hyungdong Shin (S'01-M'04-SM'11) received the B.S. degree in electronics engineering from Kyung Hee University, Korea, in 1999, and the M.S. and $\mathrm{Ph} . \mathrm{D}$. degrees in electrical engineering from Seoul National University, Korea, in 2001 and 2004, respectively. During his postdoctoral research at the Massachusetts Institute of Technology (MIT) from 2004 to 2006, he was with the Wireless Communication and Network Sciences Laboratory within the Laboratory for Information Decision Systems (LIDS). In 2006, Dr. Shin joined Kyung Hee University, Korea, where he is now an Associate Professor at the Department of Electronics and Radio Engineering. His research interests include wireless communications and information theory with current emphasis on MIMO systems, cooperative and cognitive communications, network interference, vehicular communication networks, location-aware radios and networks, physical-layer security, molecular communications. Dr. Shin was honored with the Knowledge Creation Award in the field of Computer Science from Korean Ministry of Education, Science and Technology (2010). He received the IEEE Communications Society Guglielmo Marconi Prize Paper Award (2008) and William R. Bennett Prize Paper Award (2012). He served as a Technical Program Co-chair for the IEEE WCNC (2009 PHY Track) and the IEEE Globecom (Communication Theory Symposium, 2012). He was an Editor for IEEE Transactions on Wireless Communications (2007-2012). He is currently an Editor for IEEE Communications Letters. 